\title{
Dynamic Fractional Frequency Reuse Diversity Design for Intercell Interference Mitigation in Nonorthogonal Multiple Access Multicellular Networks
}

\author{
Kashif Mehmood $\mathbb{D}^{1},{ }^{1}$ Muhammad Tabish Niaz, ${ }^{2}$ and Hyung Seok Kim $\mathbb{D}^{1}$ \\ ${ }^{1}$ Department of Information and Communication Engineering, Sejong University, Seoul, Republic of Korea \\ ${ }^{2}$ Smart Device Engineering, Sejong University, Seoul, Republic of Korea \\ Correspondence should be addressed to Hyung Seok Kim; hyungkim@sejong.edu
}

Received 16 March 2018; Revised 7 June 2018; Accepted 25 June 2018; Published 15 July 2018

Academic Editor: Kostas Peppas

Copyright (c) 2018 Kashif Mehmood et al. This is an open access article distributed under the Creative Commons Attribution License, which permits unrestricted use, distribution, and reproduction in any medium, provided the original work is properly cited.

\begin{abstract}
Nonorthogonal multiple access (NOMA) is one of the few promising techniques that can ensure the achievement of benefits foreseen in next-generation $5 \mathrm{G}$ wireless networks and beyond. By using superposition coding, NOMA allows multiple users to share the same time and frequency resources, thereby enhancing user connectivity, spectral efficiency, and a considerable increase in user throughput. Interference mitigation is an important consideration in NOMA and is considerably more influencing in multicellular environments. First, a brief description of the impairments that can arise in a NOMA cellular network along with responsible factors is provided. Second, different approaches adopted to minimize these impairments are discussed. Finally, a possible solution is proposed that consists of a coordinated approach between the individual cells in the NOMA domain to minimize interferences and improve user throughput. Adaptive fractional frequency reuse (FFR) is used to allocate distinct frequency resources to edge users of different cells to minimize intercell interference in NOMA. Simulation results prove that the proposed NOMA scheme plays an important role in minimizing impairment effects and enhancing the SINR and the throughput performance of edge users while ensuring fairness in its design.
\end{abstract}

\section{Introduction}

Wireless cellular networks have seen unprecedented growth in the last decade in terms of increasing demand for user data rates as well as massive connectivity for users. Multimedia applications and services have seen a gradual and expected increase, leading to the design of specific standards with a focus on seamless and smooth user experience. Nextgeneration wireless networks, including Long-Term Evolution (LTE \& LTE-A) were designed considering the growing user capacity needs and efficient use of the available spectrum to accommodate these users. LTE only offers a couple of fold improvement in user capacity over third-generation (3G) networks and will be insufficient, considering the expected growth.

Orthogonal multiple access (OMA) has been used widely in current and previous generations of wireless cellular networks for user access. Frequency resources are allocated in a disjoint manner to minimize interuser interference, thereby maximizing user throughput and connectivity up to a certain limit as allowed by the availability of frequency resources. Multiple OMA techniques currently being used include frequency-division multiple access (FDMA), timedivision multiple access (TDMA), code-division multiple access (CDMA), and orthogonal frequency-division multiple access (OFDM).

One of the most important and challenging criterion for next-generation $(5 \mathrm{G})$ cellular networks is for them to be able to provide user throughput $1000 \times$ more than that of current $4 \mathrm{G}$ network deployments. To fulfill these requirements, nonorthogonal multiple access (NOMA) with a successive interference cancellation (SIC) receiver was presented as one of the several promising candidate radio access techniques for future cellular networks. OMA users are separated based on a resource division mechanism, whereas a resource sharing approach is adopted for NOMA schemes. Resource sharing 
is accomplished by using the superposition principle where a composite signal is constructed from individual user signals and mapped onto a common frequency resource as opposed to one-to-one user resource mapping in OMA. NOMA provides massive connectivity as well as throughput enhancement obtained by sharing a single resource by multiple users [1]. Spectral efficiency is an embedded advantage of NOMA over OMA schemes provided by the superposition of users over a common resource.

In NOMA, user clustering is performed to pair users with diverse channel responses together to maximize user throughput gain and user capacity [1]. Another added advantage of clustering process is the simplification of SIC at the receiving end. All paired users are then mapped onto orthogonal frequency resources to avoid interference between user clusters, which is also known as intercluster interference. Interference effectively reduces the benefits offered by NOMA over OMA. Interference experienced can be due to a number of factors. Firstly, incorrect channel state information (CSI) causes errors during SIC decoding at the NOMA receiver for one or more users, depending on the user who reported the wrong CSI. Then, clustering method can lead to the wrong pairing of users causing errors in the SIC process. Also, user density in a cluster affects the complexity of SIC and the throughput limit of each user, and, finally, the number of clusters defines the amount of bandwidth available for each cluster and eventually individual user throughput gains.

For a multicell NOMA network, another major source of interference that occurs between different clusters of adjacent cells is known as intercell interference (ICI) which will be considered in the proposed work. For the multicellular wireless network, the available spectrum is allocated amongst different clusters in a cell, and spectral efficiency, as well as user capacity enhancement, is achieved by employing the frequency reuse scheme [2]. NOMA can offer significant improvements, but only if ICI and cluster interferences are managed efficiently. In this paper, ICI isolation is achieved by frequency reuse diversity along with the efficient design of user clustering as well as efficient resource utilization amongst NOMA users.

1.1. Related Work. In recent years, many NOMA schemes have been devised such as interleave-division multiple access (IDMA) [7], low-density spreading CDMA (LDS-CDMA) [8], pattern-division multiple access (PDMA) [9-14], sparsecode multiple access (SCMA) [15-17], multiuser sharing access (MUSA) [18-20], bit-division multiplexing (BDM) [21], low-density spreading OFDM (LDS-OFDM) [22], resource-spread multiple access (RSMA) [23, 24], interleavegrid multiple access (IGMA) [25], multiuser bit-interleaved coded modulation with iterative decoding (MU-BICM-ID) [26], and power domain nonorthogonal multiple access (PDNOMA) [5, 27-32].

PD-NOMA is considered in which users are distinguished on the basis of allocated transmit power on a shared resource [28]. Power allocation in NOMA is carried out considering the detection process via SIC at the receiver; hence, a user with a poor channel gain, i.e., cell edge (CE) user, is allocated a higher power and a lower power is assigned to the user with a better channel gain, i.e., cell center (CC) user [28]. CE users will be the ones most affected by the ICI as they will have to cancel out any possible spillover signals intended for CE users of adjacent cells. Higher powers are allocated to CE users to ensure throughput as well as sufficient received power at the base station (BS) for detection [28]. Both CC and CE users are then mapped onto respective frequency resource blocks (RBs). The NOMA receiver employs simultaneous multiuser detection (MUD) to identify and estimate each user's data, eliminating any effects of channel distortion or interuser interference with SIC [27]. SIC is a technique used to successively extract a single user signal by the cancellation of unwanted user signals in the received signal. SIC performs optimally when each user experiences a channel (i.e., resource block) that is considered distinct from other users [1]. However, a rapidly changing channel itself poses a problem of employing a complex channel estimation algorithm to find the channel response for each user. This feature makes NOMA a suitable candidate for outdoor cellular networks as the path loss varies considerably in outdoor scenarios as compared to indoors.

FFR [2] and soft frequency reuse (SFR) [33] are two studied solutions employing frequency reuse to improve spectrum efficiency and to reduce potential ICI in $4 \mathrm{G}$ networks. The available spectrum is divided into subchannels that are further divided into cell center and cell edge groups. By allocating different power levels to users in each group, ICI can be controlled effectively. FFR provides a high network throughput as well as a high edge user SINR as compared to SFR, which provides a balance between resource efficiency and interference reduction. The major difference between FFR and SFR is that in SFR cell center users can also use the spectrum allocated to cell edge users. This causes considerably more interference to both center and edge users when compared with the FFR case. This makes FFR a considerably better choice when interference reduction is of prime importance. In downlink OFDMA, system performance is usually limited by ICI; the edge users are the ones most affected by it. FFR was explored as a possible solution in $[2,33]$ to address the performance issues of edge users due to interference. Edge zones in cells are assigned a larger reuse factor in this scheme to reduce interference. In static FFR, the reuse factor was decided at the time of frequency planning of a network; this is inefficient and does not take into consideration the changing conditions that affect the edge zones of cells. Therefore, by using static FFR, a satisfactory system performance for edge users cannot be achieved in real environments due to the ever-changing channel and interfering conditions. This drawback of static FFR has also been highlighted in [34], which studied a dynamic adaptive frequency-division algorithm to improve cell average throughput, especially the edge user throughput for OFDMA. The results achieved by this scheme are, however, restricted to scenarios in which a single user is mapped onto an orthogonal frequency resource and no superposition coding is taking place. Detailed analysis of existing schemes for NOMA intercell interference management has been performed in 
Section 2. Hence, there is a need to develop a new scheme for ICI minimization for NOMA multicellular environments, and the prime focus of our work is to address this need.

1.2. Contribution. As already discussed, the ICI mitigation scheme introduced for OFDMA systems [34] cannot be applied to a NOMA scenario due to the fundamental differences in the multiaccess approach for users. Power allocation diversity exists for NOMA users and is the basis of differentiating users in power domain. Using SIC calls for a consideration of user clustering as well as the efficiency of the SIC process. User clustering performed to reduce the complexity and latency of the SIC process causes an overload of users on a single resource block (RB). These factors are a driving force in developing a strategy by using the advantages of frequency reuse diversity for ICI mitigation in NOMA multicellular networks. FFR is used to partition the system bandwidth into the center and edge bands and channel allocation to the respective user will be performed by the band, starting from edge users. A combined power and frequency allocation design are proposed that ensures maximum user performance for both edge and center users by allocating more power and frequency channels, respectively. The adaptive nature of power and channel allocation as per fairness criteria ensures service to edge users before center users. A novel ICI mitigation approach is proposed that includes the implementation of FFR by cell division followed by user classification into clusters and then resource allocation. A detailed discussion of the proposed design along with its implications will be performed in Section 4. The following contributions are made in this paper.

(i) An FFR-based user clustering technique for NOMA user distribution is proposed that starts with cellular segmentation as per the discussed criterion followed by user classification as CE or CC. Fairness is considered while servicing these users to ensure symmetric service to all users in the cellular service area as well as reaping the benefits of NOMA.

(ii) A dynamic power and frequency allocation scheme for NOMA users with proportional fairness for CC and $\mathrm{CE}$ users is proposed. CE users are prioritized while allocating resources to meet fairness criteria since CC users have better service and channel conditions as compared to CE users.

(iii) An FFR-based interference coordination scheme is proposed which makes use of the NOMA for providing user access to the network in dense multicell networks and meeting the guaranteed minimum service requirement for weak users in the network.

(iv) A detailed analysis is performed depicting the advantages offered by NOMA over OMA and the significance of the frequency diversity technique used for catering ICI. User throughput analysis is performed to prove the benefits of the proposed scheme with a focus on selection criterion for cell segmentation and its impact on NOMA as well as ICI experienced by CE users. (v) It is proven by simulation results in Section 5 that the proposed scheme isolates edge users of neighboring cells effectively in a multicellular NOMA environment to reduce experienced ICI. The resource allocation scheme considers the user's fairness criteria and enhances NOMA capacity as well as the throughput for $\mathrm{CE}$ as well as the CC users.

(vi) A comparison is performed with existing ICI management schemes (see Section 2) leading to a better understanding of focused role and advantages of the proposed design.

The rest of the paper is organized as follows. In Section 2, different approaches adopted for the mitigation of ICI are discussed along with any related works using that approach. In Section 3, the system model for NOMA and FFR design for analyzing the proposed scheme is described. In Section 4, the proposed adaptive FFR-based ICI mitigation technique for a NOMA multicellular environment is described in detail. Section 5 presents the simulation design for verifying the proposed scheme, and performance results are discussed in comparison to proposed goals. Concluding remarks along with possible future extensions and improvements of our work have been discussed in Section 6 .

\section{Interference Mitigation Approaches}

Interference plays a significant part in influencing communication system design as well as robustness. Major interfering sources include natural factors, which influence channel behavior like fog, rain, and pollution and channel specific factors like user density as well as the clutter differences of the covered areas. Channel estimation techniques have been developed for a different channel and area types, but all they can give are the instantaneous estimates. Here, we consider the interference between users of different cells using the same channel, i.e., ICI. Therefore, interference management (IM) is considered a critical part of a robust communication system design. IM includes three main categories of handling unwanted interference: (i) interference avoidance (IAv), (ii) interference coordination (ICo), and (iii) interference cancellation (ICa). Interference avoidance (IAv) is considered to try to isolate the interfering entities from the intended users. Interference coordination (ICo) includes the design of a coordinated design to control resource allocation with an objective of minimizing the unintended user signals. Interference cancellation (ICa) takes an approach to cancel out the interfering parts of the received signal. IAv cannot be applied in NOMA design due to the sharing of resources amongst multiple users. Proposed design consists of a coordinated design where NOMA users are allocated resources (channel or RBs and power levels) with a focus on interference minimization.

In a multicellular scenario, the received signal becomes even more complex due to the superposition of CE user signals of different cells. This makes interference removal methods and channel modeling schemes used in single-cell networks inapplicable. IM schemes are in use since long in wireless networks and following is the brief discussion 


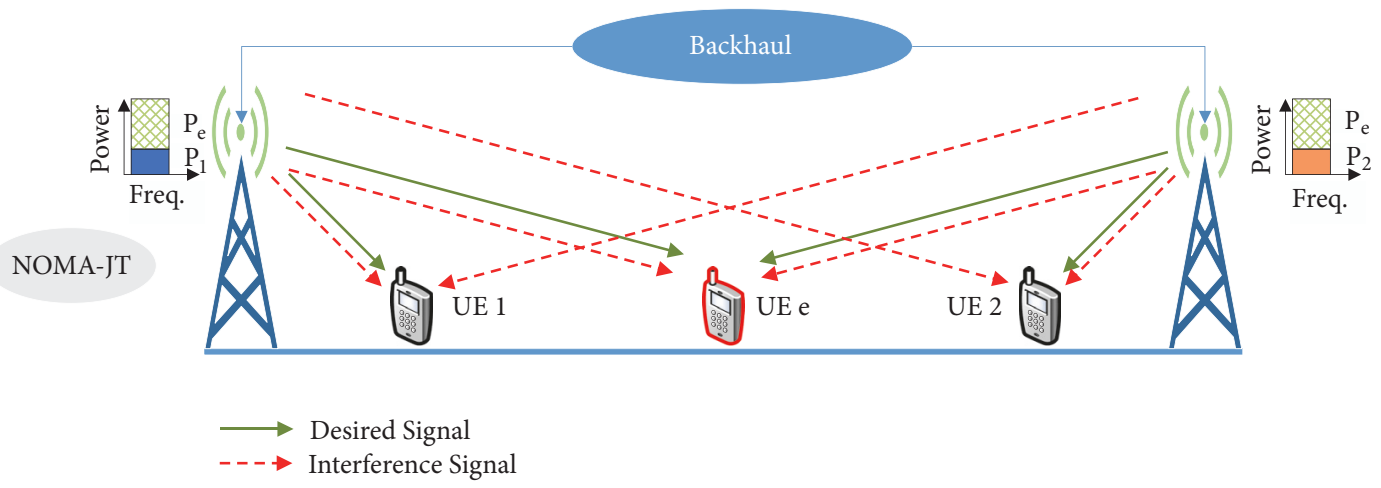

(a)
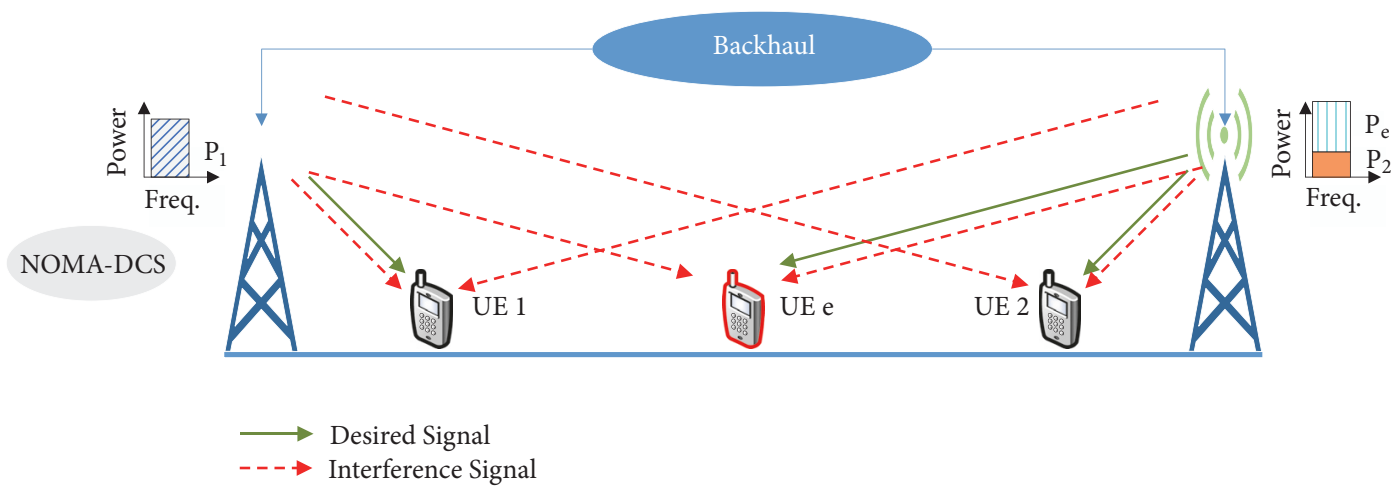

(b)

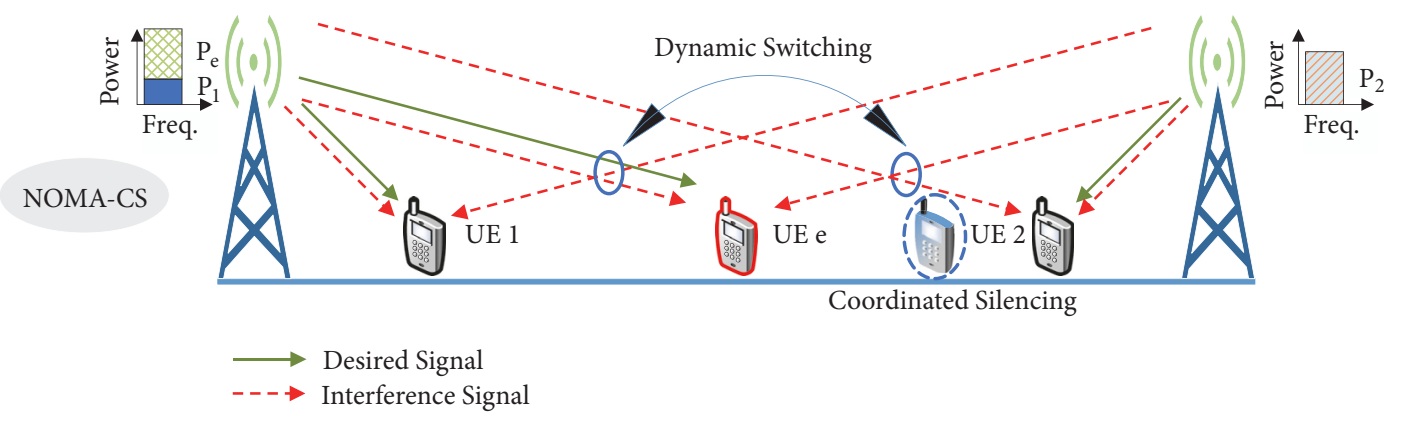

(c)

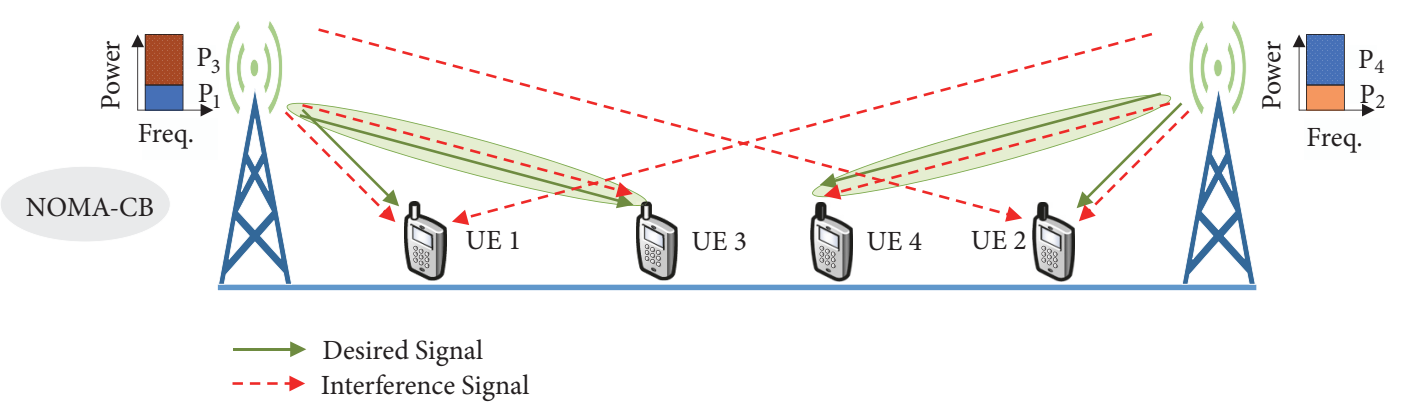

(d)

FIgUre 1: Multicell NOMA ICI mitigation solutions: (a) NOMA-JT, (b) NOMA-DCS, (c) NOMA-CS, and (d) NOMA-CB.

of those schemes when applied to NOMA. ICI causes a significant decrease in CE user performance in multicell architecture as compared to single-cell design. In this section, recent research efforts that combine IM approaches with multicell NOMA are discussed. Figure 1 shows some of the interference coordination scenarios in a multicell network. ICI is the main issue in multicell NOMA networks, as it reduces a cell edge user's performance. Multicell techniques 
are used to harness the effect of ICI. These techniques can be broadly categorized as coordinated processing $(\mathrm{CP})$ and joint processing (JP) [6]. This classification is based on whether the data messages desired by the users should be shared amongst multiple BSs or not. A single BS serves a user at any given moment in the case of the CP, whereas, in the JP, multiple BSs service a single user.

2.1. Joint Processing (JP). In NOMA-JP, user data is shared amongst multiple BSs before the user gets its data from one or multiple BSs at the same time. Two different approaches are usually considered here, namely, joint transmission (JT) and dynamic channel selection (DCS). JT is a technique in which multiple BSs serve a user simultaneously instead of disrupting each other and DCS is a technique in which multiple BSs have data for the user but only one of them serve the user at a time. In NOMA-JT, edge users receive and process signals from multiple BSs and interference can be effectively cancelled along with improvement in edge user rates. It gives the effect of a MIMO transmission as a transmission as a single user is receiving from multiple different transmitters and ICI can be effectively cancelled as in the single-cell MIMO approach [5]. A drawback of this technique is the CSI sharing overhead that should be accurately available on all transmitters. A coordinated superposition coding (CSC) scheme is detailed in [3], which eliminates the CSI overhead altogether. This coordination between cells provides CE users with a sufficient transmission rate without any effect on CC user rates. In NOMA-DCS, despite user data availability at multiple BSs, selected BS provides service to CE users, whereas the CC users are served unaffected by the corresponding BS. This eliminates ICI as only one BS's signal has user data; hence, CE users will consider signals from other BSs as only noise. It simplifies the detection process, but coordination is required between BSs for this scheme to work effectively. The selection of a serving BS will be based on channel conditions amongst other factors. Joint processing schemes require backhaul coordination amongst different candidate and serving BSs of the network to decide the transmission mode as well as the sharing of CSI information for aiding the decision. Signaling overhead makes these techniques inefficient to implement in next-generation networks.

A general architecture of JP techniques for a two-cell NOMA design is shown in Figures 1(a) and 1(b) where edge users are serviced jointly by both BSs (JT) or by a single selected BS (DCS) as per selected mode of operation. In JT mode, multiple BSs can use Alamouti coded [4] signals to transmit simultaneously to edge users to enhance performance as well as throughput. Center users will be transmitted their required signals as it is without any degradation due to joint transmission to edge users. Figure 1(a) shows the discussed design where the edge user $U E e$ is being served by two BSs jointly whereas UEs 1 and 2 are center users being served individually by respective BSs. Similarly, for DCS mode, a single BS will be selected based on the mentioned criteria to serve the edge UE while signaling backhaul is used to intimate the network and other neighboring BSs of the selection decision. This has been depicted in Figure 1(b) with only one BS serving the edge user.
2.2. Coordinated Processing (CP). In NOMA-CP, user data is only available at one BS and is not shared amongst multiple BSs although network information and CSI are usually shared for coordination. Two different approaches can be applied when $\mathrm{CP}$ is used: coordinated beamforming (CB) and coordinated scheduling (CS). In CB, data are available at only one BS and the beamforming (BF) decision is made on based on global CSI, which must be accurate, and this poses a possible drawback. In [35], a possible solution is proposed in which joint optimization of BF vectors for BSs is performed such that there are no ICI and intercluster interference. An interference alignment (IA) based CB algorithm is proposed that uses only edge user channel information and as the number of users increases, ICI is minimized without the need for any CSI. An interference channel alignment based algorithm is also mentioned, but it requires CSI information to operate. In CS, different BSs communicate with each other to serve NOMA users with low ICI, thereby ensuring proper service to $\mathrm{CE}$ users. Only one of the coordinating BS will transmit a composite NOMA signal to both its CE and CC users, whereas the other BSs will only serve their CC users by sending their intended signals only instead of a composite signal. To the best of our knowledge, no prior work has been done utilizing CS approach with respect to multicellular NOMA networks.

Figures 1(c) and 1(d) depict a CP based transmission and interference mitigation approach for CS and CB designs where a coordinated approach is adopted for CS and a directed $\mathrm{BF}$ is done to edge users of BSs, respectively. In $\mathrm{CB}$ mode, BF will be done for $\mathrm{CE}$ and $\mathrm{CS}$ users with different precoding design and $\mathrm{BF}$ vectors in order to satisfy minimum ICI for CE users. Edge users UE 3 \& 4 will be isolated in Figure 1(d) from each other's beams due to design specifications already selected to minimize the experienced ICI. In CS mode, depending on the channel conditions as well as the ICI experienced by CE users, edge users will be served via NOMA or traditional OMA techniques. In Figure 1(c), $U E c$ was receiving neighboring $\mathrm{BS}$ signals as well before coordinated silencing was activated. Interfering neighboring BS will now only serve its CC users and ICI to UE $c$ will be minimized as a result. A challenging task here will be the selection of users to be scheduled by each BS from the set of all registered users which is an NP-hard optimization problem.

These schemes were originally detailed for LTE and LTEA networks, which have been modified to accommodate changes in NOMA schemes. Figure 1 shows some of the interference coordination scenarios in a multicell NOMA network. For better understanding, a comparison of user capacity amongst these schemes is also shown in Table 1, where a two-cell architecture is considered in which each user and BS has $T$ antennas. There are also $T$ user clusters in the proposed NOMA setup. From [36], it is known that NOMA can already support $2 T$ users, whereas OMA only supports $T$ users.

The major disadvantage of utilizing joint and coordinated transmission schemes for ICI mitigation is their inherent dependency on accurate CSI as well as user channel allocation information. This emphasizes the need for accurate acquisition along with efficient channel allocation for acquiring 


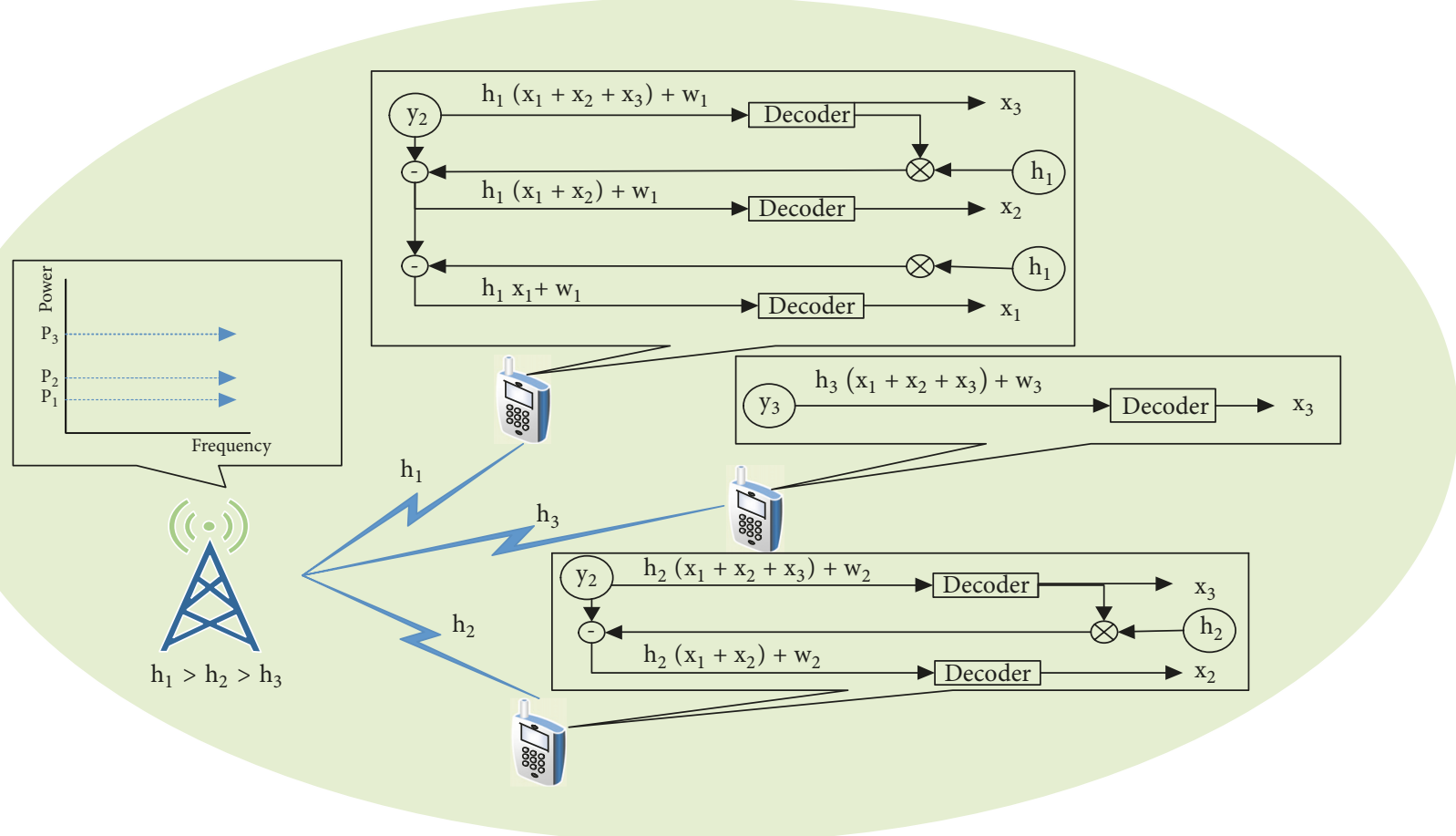

FIgURE 2: Single-cell NOMA network.

TABLE 1: Multicell noma ici techniques.

\begin{tabular}{lcccc}
\hline & $\begin{array}{c}\text { NOMA- } \\
\text { CS }\end{array}$ & $\begin{array}{c}\text { NOMA- } \\
\text { CB }\end{array}$ & NOMA-DCS & NOMA-JT \\
\hline $\begin{array}{l}\text { Transmission } \\
\text { points }\end{array}$ & 1 & 1 & 1 (selectable) & $\geq 2$ \\
$\begin{array}{l}\text { Shared } \\
\text { information }\end{array}$ & $\begin{array}{c}\text { CSI, } \\
\text { scheduling }\end{array}$ & CSI, BF & CSI, data & $\begin{array}{c}\text { CSI, data, } \\
\text { BF }\end{array}$ \\
$\begin{array}{l}\text { Backhaul } \\
\text { Type }\end{array}$ & Non-ideal & Non-ideal & Ideal & Ideal \\
$\begin{array}{l}\text { Number of } \\
\text { supported } \\
\text { users }\end{array}$ & $<4 T$ & $4(T-1)$ & $3 T$ & $3 T$ or $4 T$ \\
References & & {$[3]$} & {$[4]$} & {$[5,6]$} \\
\hline
\end{tabular}

maximum benefits from discussing approaches. CSI cannot always be estimated accurately for all the users or BSs which highlights the need for an alternate solution to the ICI problem with minimum or no depending on the channel state. Efficient channel estimation techniques are needed to fully utilize the advantage offered by discussing schemes. Discussed schemes either require a large amount of data cooperation between users or a need for an accurate synchronization of channel state as well as task coordination between users. This can become traffic intensive for cellular networks, so a novel technique is required, which guarantees performance enhancement as well as interference minimization for NOMA users to extract maximum benefits over OMA. In Section 4, a new FFR-based ICI minimization and avoidance scheme is proposed which serve as the required alternative to the discussed schemes.

\section{System Model}

3.1. Single-Cell NOMA. Consider an n-user downlink NOMA system and assume that all users experience different channel responses. The BS transmits $n$ different superimposed signals, which are multiplexed nonorthogonally in the power domain for each user using a single shared frequency resource as shown in Figure 2. Each user receives the composite signal consisting of all user signals and extracts its own signal using SIC. A user classifies all signals except its own as interference and cancels them out before retrieving its own signal. There must be a considerable separation between user signals so that SIC is able to separate and decode signals for all users. This is ensured by the power allocation scheme in NOMA that allocates power levels accordingly. Users near the BS are allocated low power levels as they will have a better channel condition as compared to far users that will experience more fading and path loss. Figure 2 shows a three-user downlink NOMA scenario with users having channel gains $h_{1}, h_{2}$, and $h_{3}$, where $h_{1}$ is the highest and $h_{3}$ is the lowest. Power allocations will be as shown with the highest power allocated to $U E_{3}$ and the lowest power allocated to $U E_{1}$ as it already has a strong channel response. This ensures that far users having a weak channel gain will receive lower interference levels from users having strong channel gains due to the lower power allocated by NOMA. Moreover, strong users will receive more interference from weak users due to higher power allocation by NOMA, but as 


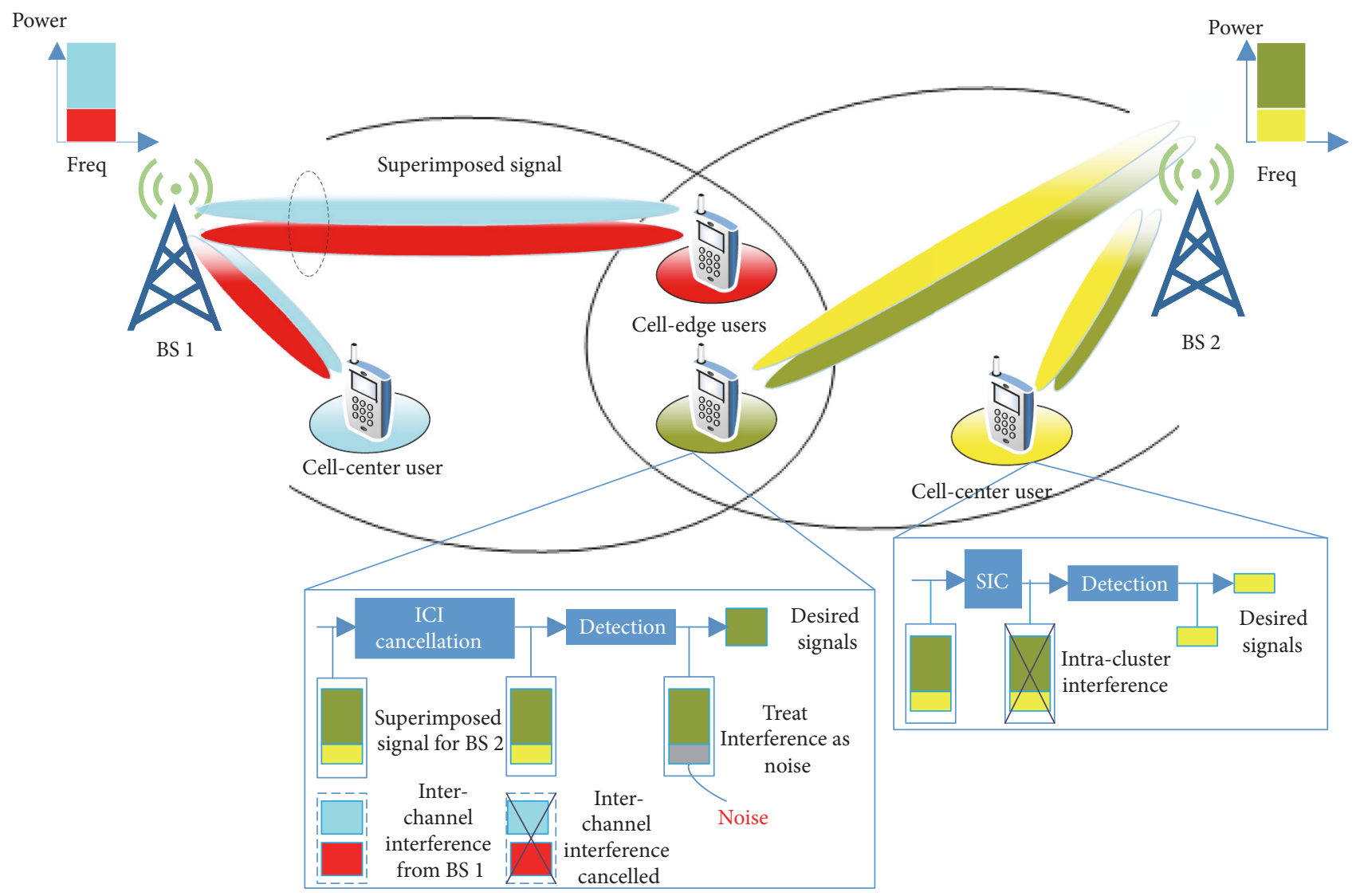

FIgURe 3: Multicell NOMA network.

strong users have a better channel condition, they will easily decode via SIC. A composite NOMA signal constructed for the network in Figure 2 is represented as

$$
x=p_{1} x_{1}+p_{2} x_{2}+p_{3} x_{3} .
$$

Now the received signal at each $U E_{i}$ can be described below as

$$
y_{i}=h_{i} x+w_{i}
$$

where $h_{i}$ is the channel response to the $i_{\text {th }}$ user and $w_{i}$ is the received noise including external and internal interferences as well.

3.2. Multicell NOMA. In this section, a multicellular downlink NOMA network and a SIC receiver design for the reception of the NOMA composite signal at each UE is modeled as depicted in Figure 3. Two types of users are defined in a multicellular setup; CC users are near the BS and $\mathrm{CE}$ users are near the boundary of cell coverage. In a multicell network, all users, especially CE users, will experience ICI irrespective of whether OMA or NOMA is used. However, in the case of NOMA, ICI is much worse as edge users will experience ICI all the time as compared to OMA in which only some time slots or frequency bands will be affected. In NOMA, a key feature is that channel difference is usually used to pair users into clusters. NOMA normally pairs users experiencing strong and weak channel responses together to ease the process of SIC. As per assumption, cell center users do not suffer from any ICI and only edge users are affected.

Consider the downlink of a multicell NOMA scenario with $N$ different cells and $K$ users in each cell. The total system bandwidth is denoted as $B$ and it will be further divided into $L$ total subbands. For simplicity, the number of receiver antennas at user terminal is taken as 1 . Each BS has a total transmission power limit of $P_{\max }$. Resource allocation for each user is performed in terms of subchannels and this gives us the benefit of multiuser diversity in the frequency domain. Now, the multiuser scheduler maps a set of users, $U_{b}=\left\{u_{b}(1), u_{b}(2), u_{b}(3) \ldots u_{b}\left(m_{b}\right)\right\}$ to a frequency block, $b(1<b<L)$. Here, $u_{b}(j)$ represents the $j$ th $\left(1<j<m_{b}\right)$ user index scheduled at frequency block $b$ and $m_{b}$ denote the total number of scheduled users at scheduled users at frequency block $b$. In the downlink, BS will channel code and modulate each user $u_{b}(j)$ th data independently of each other. The available signal, $x_{b}$, at a frequency resource $b$ is the sum of $u_{b}(j)$ th coded modulation symbol $s_{b}\left(u_{b}(j)\right)$. Therefore, $s_{b}\left(u_{b}(j)\right)$ of all $m_{b}$ users is a superposition expressed as

$$
x_{b}=\sum_{j=1}^{m_{b}} \sqrt{p_{b}\left(u_{b}(j)\right)} s_{b}\left(u_{b}(j)\right) \text {, }
$$

where $E\left[\left|s_{b}\left(u_{b}(j)\right)\right|^{2}\right]=1$ and $p_{b}\left(u_{b}(j)\right)$ is the power level assigned to user $u_{b}(j)$ for transmission at frequency block 
b. In Figure 3, CE users will have more interference as well as more signal fading and hence a larger portion of available power in the composite signal must be allocated to them for meeting performance goals. Consequently, center users will be allocated less power as compared to edge users because center user signals will have less fading impact as compared to edge users. The received signal for each user $u_{b}(j)$ at a frequency block $b, y_{b}\left(u_{b}(j)\right)$, is shown as

$$
y_{b}\left(u_{b}(j)\right)=h_{b}\left(u_{b}(j)\right) x_{b}+w_{b}\left(u_{b}(j)\right),
$$

where $h_{b}\left(u_{b}(j)\right)$ and $w_{b}\left(u_{b}(j)\right)$ are the channel response and the noise plus ICI contributions in the received power, respectively, experienced by user $u_{b}(j)$ at frequency block $b$. Channel coefficients modeled as propagation loss, shadowing loss, and instantaneous fading loss coefficients will be kept constant within a frequency block. ICI is treated by the SIC receiver as white noise and it performs maximum ratio combining (MRC) on received combined signal $y_{b}\left(u_{b}(j)\right)$ as

$$
\begin{aligned}
\dot{\hat{y}}_{b}\left(u_{b}(j)\right) & =\frac{h_{b}^{H}\left(u_{b}(j)\right) y\left(u_{b}(j)\right)}{\left\|h_{b}\right\|} \\
& =\sqrt{g_{b}\left(u_{b}(j)\right)} x_{b}+z_{b}\left(u_{b}(j)\right),
\end{aligned}
$$

where $g_{b}\left(u_{b}(j)\right)=\left\|h_{b}\left(u_{b}(j)\right)\right\|^{2}$ and $z_{b}\left(u_{b}(j)\right)$ are the equivalent channel gain and noise plus ICI after MRC, respectively. The average power level of channel gain is denoted as $n_{b}\left(u_{b}(j)\right)=E\left[\left|z_{b}\left(u_{b}(j)\right)\right|^{2}\right]$. For NOMA signal reception, each UE implements SIC to recover its individual signal from the superposed received signal. The decoding order for SIC depends on the ratio between the channel gain and the interference seen by each user that includes noise and ICI as already discussed. Hence, NOMA user will in sequence detect signals of all those users whose turn comes before decoding its own individual signal from the composite signal.

If ICI can only be experienced from adjacent cells by either a CC or CE user in the $i_{\text {th }}$ cell and no interference is encountered via SIC or other clusters in a cell, the signalto-interference-plus-noise ratio (SINR) for NOMA users on frequency block $b$ is calculated as

$$
\operatorname{SINR}_{j}=\frac{\left(1 / m_{b}\right) \sum_{i=1}^{m_{b}} y_{b}^{2}\left(u_{b}(i)\right)}{\sum_{u_{b}(i) \epsilon U_{b}} y_{b}^{2}\left(u_{b}(i)\right)+w_{b}^{2}\left(u_{b}(j)\right)} .
$$

For NOMA users in a cluster, the achievable user rate for each user $U E_{i}$ can be represented as [1]

$$
\dot{R}_{i}=\mu L \log _{2}\left(1+\frac{P_{i} h_{i}}{\sum_{j=1}^{i-1} P_{i} h_{i}+\mu}\right)
$$

where $\mu$ is the number of channels assigned to the user and $L$ is the bandwidth of each channel.

\section{Proposed Joint ICI Minimization \& Resource Allocation Scheme}

Frequency reuse schemes have since long been used to enhance user capacity and for efficient use of the allotted frequency spectrum. Frequency reuse has also found its applications for the minimization of interference between adjacent cells, ensuring better performance for edge users as they are the prime victims of this interference. FFR and SFR are two possibilities when using frequency reuse diversity to cancel ICI [37]. As previously discussed, FFR is more effective in minimizing ICI because of its isolation of channels for edge and center users, so it will be used in the proposed solution instead of SFR [2, 33]. SFR takes precedence over FFR (or strict FFR) due to its greater resource efficiency due to the sharing of resources amongst CE and CC users [38].

Diversity in frequency reuse is used for the minimization of ICI and different reuse factors are used for center and edge zones of cells [37-39]. Frequency isolation is established by using a higher reuse factor in edge zones of cells in a multicellular environment. Orthogonality is achieved by using FFR in NOMA and this can also be known as an OMA over NOMA system where a feature inherent to orthogonal access for eliminating interference is used. Static FFR was first proposed in which fixed frequency and power allocations were made to the edge and center users where a changing channel will result in ICI. Hence, an adaptive FFR scheme is needed that will allocate power and frequency resources to the respective cell center and edge users depending on inherent channel conditions. FFR is implemented along with NOMA by firstly dividing each cell into edge and center regions and then allocating resources, including power and spectrum to those resources. User clusters are formed to group users together in respective zones to reap the benefits of NOMA as shown in previous works. Cell zoning leads to a degradation in performance of the proposed NOMA due to the division of resources, but this is considered as a tradeoff in the proposed work. In the case where ICI mitigation is not performed, considerable performance degradation has been observed when compared with the cases in which ICI mitigation is performed. The proposed design is suboptimal in the sense that it tries to improve the interference cancellation performance of proposed scheme based NOMA better than conventional NOMA in return for slightly reduced performance benefits of NOMA. Another added benefit of the proposed scheme is a need for low user coordination as well as the accuracy of CSI information at respective users.

A multicell FFR scheme has been shown in Figure 4 where the edge and center zone division has been done along with bandwidth allocations to each zone. A fraction of the band $\left(B_{C}\right)$ has been allocated to users in central zone with a reuse factor of 1 . Edge zones have been allocated a fraction of the band from the edge user band with a reuse factor of 3 to avoid ICI with neighboring cells. NOMA power allocation factor for edge users will be higher as compared to center users to compensate for the reduction in bandwidth allocation. Due to the separation of the band for edge users of neighboring cells, ICI power will decrease whereas signal power will increase due to higher power allocation leading to an improved SINR for edge users for proposed NOMA-FFR based ICI minimization scheme. Salient features of proposed scheme have been discussed in detail in this section. 


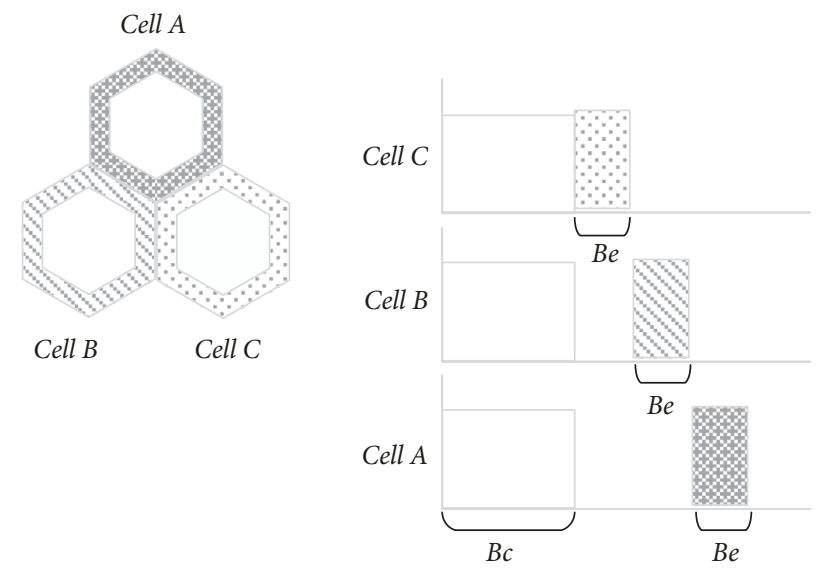

Figure 4: Fractional frequency reuse (FFR).

4.1. Cell Zoning. As discussed, NOMA clusters are made in such a way that users with the highest and lowest channel gain are paired together as CSI diversity improves SIC and NOMA performance. However, in this case, we will pair users a little broader as edge and center users by partitioning each NOMA cell. A user categorization method is devised for categorizing available cell users into near and far zones. It will help us in understanding the effects of ICI on these different user types. One of the important parameter to consider in this regard is the radius of coverage for center users, denoted as $r_{c}$, which defines the boundary of the near (central) region of the cell as per consideration. To categorize users as center or edge, an estimate of their distance from the transmitter is required in each cell which can be difficult to acquire accurately in practical scenarios. Instead, we will use two different approaches.

(i) Received SINR from the serving cell is compared to threshold SINR value.

(ii) Difference between received powers from serving and neighboring cells is compared to a threshold power level.

These techniques represent a composite user classification criterion that will be used to classify users in each cell of our network as CE or CC. Due to the difficulty in distance measurement between each individual user and cell center, SINR is instead used. SINR at any given distance from the serving cell is directly dependent on the distance between the user and the transmitting source, and therefore it can be used instead of distance to categorize users. We define a total of $J$ interfering adjacent cells for each cell and for each user either of the above-mentioned approaches are used to categorize them into cell zones depending on the amount of ICI experienced. For each user, we have a serving cell and an arbitrary number of adjacent interfering cells. SINR for each user is represented as (6) and we can define the abovementioned approaches for the $k^{\text {th }}$ user in each i-th cell as

$$
\begin{array}{r}
\left(S N I R_{i}\right)^{k}-\sum_{j=1}^{J}\left(S_{N I R_{j}}\right)>S_{T H} \\
\left(S_{N I R_{i}}\right)^{k}>S_{T H},
\end{array}
$$

where $S_{\mathrm{TH}}$ is defined as the decision threshold for the division of cells into edge and center zones and it depends on the CSI as well as the user density in each cell. Users near the cell center experience almost negligible ICI and the second term in (8a) is close to zero, which gives us only the SINR of each user to be used for comparison. We can use (8a) for CE as well as CC users, but as ICI effects are minimal for CC users, (8b) can be used instead for simplicity. The average SINR of every user is calculated and categorization is performed accordingly as near users will have a better SINR as compared to far users. Now, each cell can label its users as a center or edge user depending on an SINR threshold already calculated to depict the channel model and conditions. Hence, users are divided into two groups: $U_{E}$ for the edge users and $U_{C}$ for the center users.

4.2. Resource Allocation. Users are considered to be uniformly distributed in the service area of a cell as is the case in most practical scenarios and as shown in [40]. After distinguishing the edge and center users, power and frequency channel allocation will take place, ensuring that user fairness is maintained across the cell for each cell in the network. In NOMA, power allocation is carried out jointly and, for a single user, it not only limits the achievable throughput of that user but all users in the NOMA cluster. We will consider the tradeoff between allocating more power, or bandwidth to users depending on their requirement and whether they are on the edge or central zone of the cell. $\mathrm{CE}$ users must be allocated more power to enable them to effectively communicate with the transmitting source. This is because they will be the ones most affected by ICI due to them being in the edge zone of the cells. CC users will be allocated lower power levels as compared to CE users because they have better channel conditions and a better SINR. Power allocation to the edge and center users is done keeping in mind that the sufficient power difference exists between them in order to ensure signal recovery via SIC at respective receivers. This can be depicted by the following condition which needs to be satisfied at each individual user:

$$
P_{i} h_{i-1}-\sum_{j=1}^{i-1} P_{j} h_{i-1} \geq P_{\max }
$$

where $P_{i}$ is the allocated power level to the $i^{\text {th }}$ user in a NOMA cluster and $h_{i}$ is the normalized channel gain experienced by that user whereas $P_{\max }$ is the power budget for NOMA cluster. As implied by (9), transmit power allocated to any user must be greater than the sum of transmit power of all users with a relatively stronger channel defined as the necessary condition for SIC decoding in NOMA systems [1]. This ensures that sufficient power separation exists within each cluster so that users can successfully distinguish each user's signal in composite received NOMA signal. It has been assumed for simplicity's sake that SIC is done perfectly with no error propagation so that focused analysis of ICI can be performed which is the prime target of the proposed algorithm.

The available frequency spectrum will be divided into cell center and cell edge zones. FFR will be applied by 
assigning $1 / 3^{\text {rd }}$ of the total spectrum $B$, denoted as $B_{E}$ to CE users and remaining $2 / 3^{\text {rd }}$ denoted as $B_{C}$ to CC users in meeting their respective throughput requirements as well for compensating ICI. All channels in the CE spectrum must always be orthogonal to the $\mathrm{CE}$ channels in neighboring cells for ICI reduction. The following parameters are defined in the resource allocation process used to ensure fairness, throughput performance, and ICI mitigation for the NOMA cellular network:

(i) Edge user minimum rate threshold $\left(\mathrm{R}_{\min }\right)$

(ii) Center user maximum rate threshold $\left(\mathrm{R}_{\max }\right)$

(iii) Maximum cell power threshold $\left(\mathrm{P}_{\max }\right)$

These parameters will ensure fair and efficient allocation of power and frequency resources to edge and center NOMA users. ICI will be eliminated by using FFR in the proposed scheme, whereas throughput for all NOMA users will be ensured by keeping a specific channel dependent rate limit on both central and edge users. Channels from the available spectrum will be allocated to edge users by considering the minimum rate requirement $R_{\min }$, which will depend on the channel conditions as well the available spectrum and power allocations. $R_{\min }$ will ensure edge users get sufficient service rates in proposed network design and it will also dictate the amount of power which will be required for each edge user in NOMA setup. Similarly, channel allocation to center users will be carried out considering the $R_{\max }$ rate, which is needed to restrict rates for center users remain within a limit when they will be operating in a NOMA mode along with edge users. As base stations have specified power levels for different modes of operation, $P_{\max }$ is defined as the maximum transmitting power that can be allocated to a NOMA user. Edge users will be allocated higher power levels in NOMA to compensate for the path loss they will endure due to larger distances as compared to center users. Power allocation to NOMA edge users will be kept under this practical limit of $P_{\text {max }}$.

For an optimal solution, the water-filling approach can be used for power allocation to center and edge users as per their channel gains. However, this would require an iterative process starting from an initial assignment of powers to all users and then gradually refining power allocation for each user. Convergence will depend on the number of users as well as the defined maximum average sum rate. This process is computationally complex and depends on knowledge of already allocated powers to users in the beginning. Alternate solutions include, firstly, the allocation of fixed power to all users depending on a fixed allocation factor, which is adjustable and users will have information about their allocated power. Secondly, the fractional power allocation approach can be used that compensates the channel variations for users with adaptive power control. The latter approach when used will make fair power allocation to users in our design possible with low complexity and user feedback.

Power is allocated to each user by using a proportional fairness (PF) based technique [41], which will make sure that the resource allocation satisfies the given constraints.
(1) Divide total bandwidth $\mathbf{B}$ into $\mathbf{B}_{\mathrm{C}}$ \& $\mathbf{B}_{\mathrm{E}}$ with a

(2) total of $\mathbf{L}$ channels.

(3) for each $u_{b}$ in $U_{b}$

(4) if $u_{b} \longrightarrow U_{E}$ do

(5) Assign a single channel.

(6) $\quad \mathrm{B}_{\mathrm{E}}=\mathrm{B}_{\mathrm{E}}-1$

(7) if $\mathrm{P}_{n, b}>\mathrm{P}_{\max }$ do

(8) $\quad$ if $B_{E} \neq \phi$ do

(9) Assign another channel to reduce

(10) required power \& meet $\mathbf{R}_{\min }$.

(11) $\quad \mathrm{B}_{\mathrm{E}}=\mathrm{B}_{\mathrm{E}}-1$

(12) else do

(13) Set total assigned power of CE user

(14) group to $\mathrm{P}_{\max }$.

(15) end

(16) end

(17) $\quad \mathrm{U}_{\mathrm{E}}=\mathrm{U}_{\mathrm{E}}-1$

(18) else if $u_{b} \longrightarrow U_{C}$ do

(19) for channels in $B_{E}$

(20) Map a center user on the same channel as

(21) an edge user.

(22) Allocate power as per $\mathbf{R}_{\max }$ and considering

(23) power allocation of edge user as well on

(24) the same channel using (10).

(25) $\quad \mathrm{P}_{\mathrm{C}}=\mathrm{P}_{\mathrm{E}}-1$;

(26) $\quad \mathrm{B}_{\mathrm{C}}=\mathrm{B}_{\mathrm{C}}-1$

(27)

(28)

(29)

(30)

(31)

(32)

end

$$
\mathrm{B}_{\mathrm{C}}=\mathrm{B}_{\mathrm{C}}-1
$$

for channels in $\mathrm{B}_{\mathrm{C}}$

Assign channel and power to remaining users from $B_{C}$ as per rate requirement $R_{\max }$. $\mathrm{B}_{\mathrm{C}}=\mathrm{B}_{\mathrm{C}}-1$

(33) end

(34) $\mathrm{u}_{\mathrm{b}}=\mathrm{u}_{\mathrm{b}}-1$

(35) End

Algorithm 1: Fair resource allocation for edge and center users.

Total transmission power allocated for each NOMA user $k$ at frequency resource $b$ in an $n^{\text {th }}$ cell is given as

$$
p_{b}(k)=\frac{P_{n, b}}{\sum_{j \in U_{b}(b)}\left(g_{b}(j) / n_{b}(j)\right)^{-\beta}}\left(\frac{g_{b}(j)}{n_{b}(j)}\right)^{-\beta}
$$

where $P_{n, b}$ and $\left(g_{b}(j) / n_{b}(j)\right)$ represent the total transmit power of all users and the channel gain for the $j^{\text {th }}$ user for frequency block $b$, respectively. $U_{b}$ is the set of users mapped to a single frequency resource. $\beta$ is the decaying factor and a value of $\beta=0$ will result in an equal distribution of power to all users irrespective of channel gains. Allocated power will decrease with the improvement in channel conditions of the NOMA users. This signifies the role of channel gain and noise along with ICI in the selection of power levels for edge and center users. Respective channel and power selection schemes are described below and shown in Algorithm 1.

4.2.1. Edge Users. Edge users are the ones most likely to be affected by ICI, so their performance is prioritized to achieve 
throughput and capacity gains which NOMA offers. In the first stage, channel and power will be allocated to edge users to meet the rate requirements as defined by $R_{\min }$ whose value will depend on channel conditions as well as a user fairness criterion. All users in edge user group $\left(\boldsymbol{U}_{E}\right)$ will be allocated a single channel and the power level will be derived from the minimum rate requirement for edge users using (7). The essential condition for the SIC process (9) must be kept in mind during power allocation. Another condition to be satisfied in this step is the bound as already defined in the form of $P_{\max }$, which is the maximum transmit power of the transmitting source in the cell. The cumulative power of all users in an $n^{\text {th }}$ cell for each frequency channel $b$ should be less than the maximum transmit power in that cell as defined below.

$$
\sum_{j=1}^{m_{b}} p_{b}\left(u_{b}(j)\right)=P_{n, b} \leq P_{\max }
$$

To ensure the validity of (11), multiple channels are allocated to edge users, so that a lower power level can be allocated for each channel. The bandwidth allocated to each edge user will therefore increase and a lower power level will be sufficient for them to meet the minimum rate condition. This decrease in power will also benefit in terms of interference reduction between cells as compared to normal conditions. Therefore, a fair resource allocation is achieved for edge users by considering $P_{\max }$ and $R_{\min }$ bounds, as well as ICI, to be reduced for all the cells in the NOMA network.

To evaluate cell edge performance, we define an instantaneous user rate for $U E_{i}$ obtained from (7) at any time instant $\mathrm{t}$ as $R_{i, t}$. Edge users will be considered in outage when $R_{i, t}<R_{\text {min }}$, so, we can define average outage probability for proposed design as

$$
P^{N F F R}=\frac{\sum_{i \in K} \sum_{j \in N} \mathbb{P}\left(R_{i, t}<R_{\text {min }}\right)}{\sum_{i \in K} U_{E}}
$$

where $\mathbb{P}\left(R_{i, t}<R_{\text {min }}\right)$ gives us the probability that an edge user will be unable to meet the minimum rate criterion as defined in the proposed resource allocation scheme.

4.2.2. Center Users. Users close to the transmitting source receive a high SNR as well as a low interfering power from ICI due to considerable path loss. After sufficient power and resource allocation to edge users, center users will be allocated resources from the cell center resource pool. A single center user is mapped onto the same channel as an edge user for maximizing capacity, spectral efficiency, and maximum throughput for that channel ensured by NOMA. For each channel, a NOMA cluster size of two is used where a CE edge user will be paired with a CC user, but this does not hold true for all cases. CC users can be paired together as well on the same channel after ICI coordination is achieved. This resource allocation to center users is performed considering the $R_{\text {max }}$ constraint as defined before and must be met in all cases for user fairness. Power allocation values to center users will be calculated by ( $8 \mathrm{a}$ ) and ( $8 \mathrm{~b})$ and allocated accordingly to ensure rate requirements. Remaining channels will be allocated to any center users left within the already described constraints.

NOMA offers user throughput and capacity enhancements through sharing of spectrum resources by multiple users [1]. However, in proposed algorithm bandwidth for edge and center users is somehow isolated. This will lead to a loss in performance advantages offered by NOMA. Interferences experienced by NOMA users will become a considerable factor for diverse cellular environments currently in deployment due to high user density and small cell sizes. This will effectively reduce the user performance benefits offered by NOMA over OMA. This is a performance tradeoff introduced when trying to minimize ICI by the proposed algorithm. Attempts have been made to compensate for the loss in performance by allocating more channels to center users or edge users and more power to edge users. This affects user performance, but will considerably compensate for the reduction in performance due to ICI experienced by edge users. A PF fairness-based scheduler is introduced which will serve the edge users on priority for meeting rate requirements.

The main focus of the proposed design is to minimize ICI by isolating edge user bands in neighboring cells in a multicell environment. An alternate scheme to deal with ICI is proposed which does not have a dependency on CSI as other schemes already discussed in Section 3. A slightly modified NOMA is implemented with OMA functionality also being used in order to cater for ICI. This will have application feasibility in dense network deployments in future generation networks like ultradense networks (UDN). Due to a massive number of users channel state will be severely affected and a hybrid approach will be needed to compensate for the introduced interferences. User performance will be compensated by the allocation of more resources (power or channels) depending on experienced interference levels as well as target rate requirements. Simulation results indicate the suitability of the proposed scheme for ICI compensation in multicell environments by employing modified NOMA scheme.

\section{Performance Evaluation \& Results}

In this section, the proposed interference minimization technique will be analyzed and the system performance will be discussed. The premise of the superiority of NOMA over OMA is proven along with the effectiveness of frequency reuse diversity as a basis for ICI mitigation in FFR design. The minimum rate requirement criterion is proven to be an effective condition in ensuring fairness in the NOMA system. Link-level simulations were performed in MATLAB with parameters given in Table 2.

5.1. Simulation Setup. A multicellular network configuration is simulated with diverse parameters to create a realistic environment. The network model consisting of 19 hexagonal cells (radius $=500 \mathrm{~m}$ ) arranged in a wraparound manner of neighbor relations is used for simulations. Users are assumed to be distributed uniformly in each cell in either its edge or 
TABLE 2: Simulation parameters.

\begin{tabular}{ll}
\hline Cell layout & 19 hexagonal cells, 3 sectors per cell \\
Cell Radius & $500 \mathrm{~m}$ \\
Shadowing factor & Log-Normal with 8-dB Std. Dev. \\
Shadowing correlation & 0.45 (intercell), 1(intracell) \\
Channel modeling & 6 -tap typical urban (TU6) \\
Path loss model & $133.6+35$ log10 (d[km]) dB \\
Thermal noise density & $-174 \mathrm{dBm} / \mathrm{Hz}$ \\
BS transmit power & $46-50 \mathrm{dBm}$ \\
System bandwidth & $20 \mathrm{MHz}$ \\
Channel bandwidth & $200 \mathrm{KHz}$ \\
No. of users & 30 per cell $(10$ per sector) \\
Sub-channels & 100 \\
$\alpha_{\text {decay }}$ (decay factor) & 0.6 \\
Frequency reuse factor & $1($ center), 1/3(edge) \\
OMA & Subcarriers $=64, \mathrm{CP}=1 / 4, \mathrm{FFT}=$ \\
Digital modulation & 64 -point \\
Maximum NOMA User & $8,16,64$-PSK \\
Clustering Order & 2 \\
\hline
\end{tabular}

central zone depending on the cell zoning boundary. Each cell has exactly 30 users who are distributed randomly in each of the cell zones with a maximum of 10 users per sector. Users in each cell will suffer intercell residue power from interfering cells from first-order neighbors only. The available spectrum of $20 \mathrm{MHz}$ is divided into subchannels of $200 \mathrm{KHz}$, each of which will be allocated to demanding users via our resource allocation algorithm. Wireless channel is assumed to be a dense urban design based on a 6-tap typical urban (TU) channel environment with Rayleigh fading. Edge users will experience ICI from neighboring cells, which is treated as noise. The distance-dependent path loss with a decay factor of 35 is experienced by all users, especially affecting edge users, along with log-normal shadowing losses with a standard deviation of $8 \mathrm{~dB}$. At the receiver, Turbo codes with a $(1 / 3)$ root are used for error correction for ensuring data integrity. Channel estimation is assumed to be ideal which is performed via pilot symbols embedded in OFDM design. Moreover, performance comparisons of the proposed NOMA based ICI mitigation scheme are performed with traditional OMA and NOMA based designs with no ICI mitigation technique. NOMA design with the proposed scheme is also compared with available ICI management techniques to supplement the performance of NOMA in medical environments.

During the network setup phase, each user selects its serving BS based on the strongest received SNR from all the communicating BSs. Each BS is then divided into a cell zone (center or edge) depending on its proximity to its BS location. A zone division distance of half the radius of each cell is used initially and is later refined depending on the SNR threshold during simulations. Users are, respectively, allocated to a cell zone depending on this zoning criterion as mentioned in the previous section. In case of NOMA, users are prepared with a cluster size of 2 for simplicity. FFR is then implemented in each cell after cell zoning has been completed. Frequency reuse factors (RF) of 1 (for center zone users) and 3 (for edge zone users) are, respectively, used to effectively represent the ICI scenario within the NOMA scheme. In simulations, only edge users are considered to be affected by the ICI; this is relatively a safe assumption considering the cell zoning process and the distance between center zones of neighboring cells. Edge and center users are then allocated appropriate resources as per the fairness criterion depicted as the conditions mentioned before. The exact values of these parameters depend on specific channel conditions and are determined analytically. Both users within a cluster are then allocated appropriate powers as per their CSI such that they can be multiplexed together using NOMA. At each UE, SIC is performed to extract its data from the superposed signal. Network simulations are then performed to confirm the benefits of the proposed scheme.

5.2. Simulation Results. To evaluate the proposed algorithm, all mentioned premises, as well as assumptions, will be analyzed. OMA and NOMA are compared to establish the superiority of NOMA performance over OMA, considering the user fairness conditions already mentioned. The impact of reuse factor diversity on user SNR is discussed to prove the effectiveness of FFR in the proposed solution to ICI. An analysis of the proposed algorithm is performed with respect to the relation between user and power ratios for the center and edge zones with a focus on throughput performance. It is concluded from our discussions and the results that the proposed NOMA design outperforms conventional NOMA in terms of interference and throughput performance.

5.2.1. Fair NOMA versus OMA. For fair NOMA, the power allocated to the center and edge users will not be fixed, but it will be carried out in such a way as to ensure symmetry in performance between the center and edge users. For edge UE, the minimum rate requirement $\left(R_{\min }\right)$ and the maximum rate requirement $\left(R_{\max }\right)$ will be considered to ensure appropriate service and fairness in NOMA design. Fair NOMA will offer a higher capacity than fixed power NOMA and OMA. However, this behavior will change as SNR increases and for considerably larger values, the capacity performance of fixed and fair NOMA approaches will be almost similar as shown in Figure 5. This behavior has also been highlighted in [42] and as the SNR approaches infinity no matter how much power is allocated to the stronger user, the capacity increase will be constant.

5.2.2. Frequency Reuse Diversity. FFR makes use of the frequency reuse concept to distinguish cell zones where different reuse factors are used for center and edge zone users to isolate ICI for edge users. Frequency reuse diversity is the key feature in enabling the proposed algorithm to effectively minimize any interference from neighboring cells. Figure 6 depicts the effects of choosing different reuse factors with respect to user SNR for the center and edge users. It clearly shows that center users having a lower reuse factor $(\mathrm{RF}=1)$ will perform 


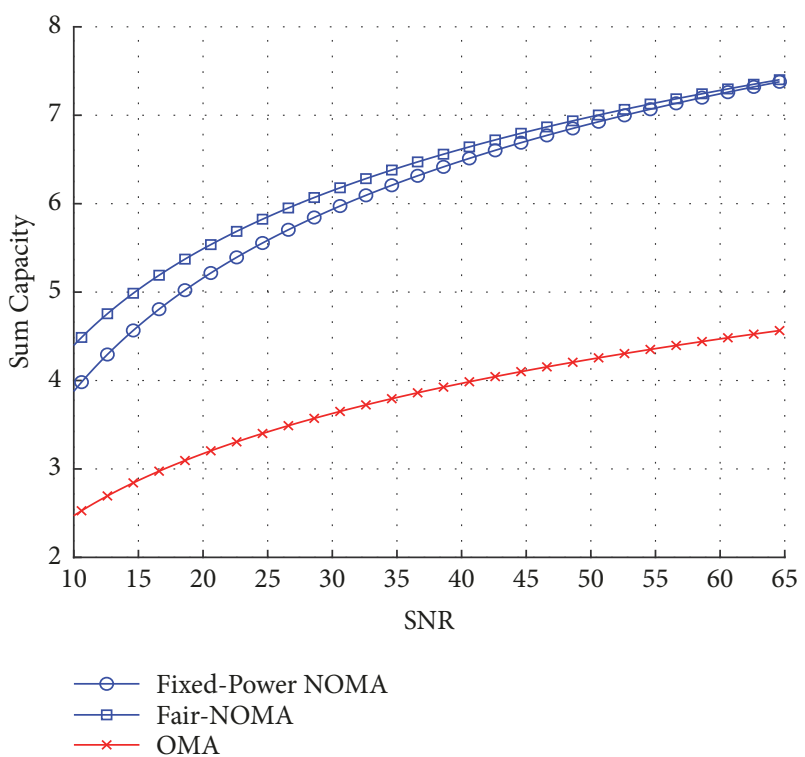

FIGURE 5: Capacity performance of fair versus fixed power NOMA.

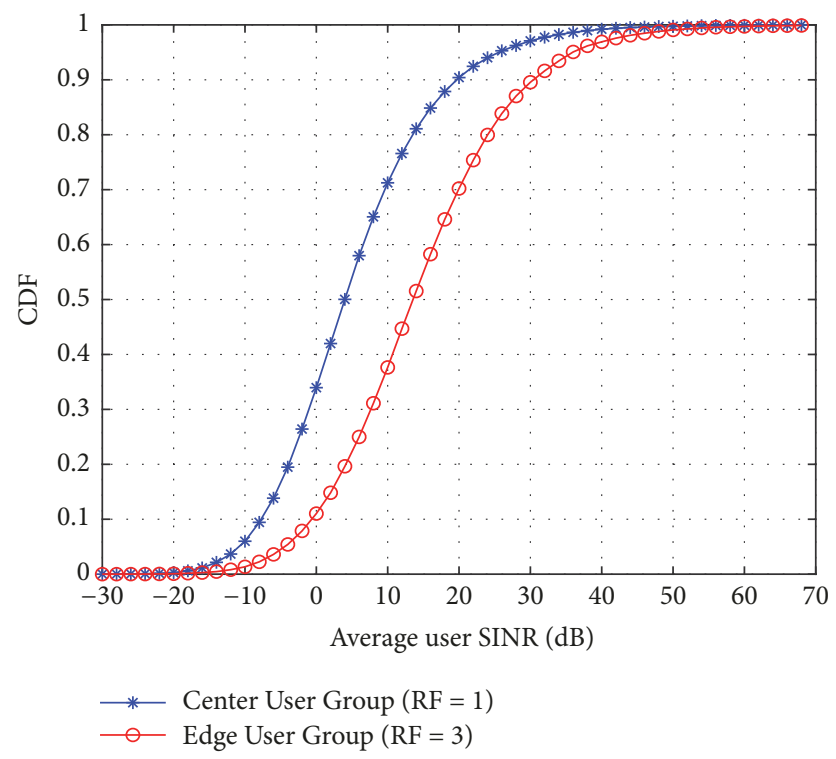

FIGURE 6: Frequency reuse diversity analysis.

considerably better than the edge users $(\mathrm{RF}=3)$. Edge users will experience higher ICI as compared to center users and this explains their distribution behavior. The dependency of received SINR by users in the center and edge zones over frequency reuse diversity has been depicted in experimental observations. Center users due to better channel conditions along with low interference factors will have higher SINRs which is shown to be $>-10 \mathrm{~dB}$ for more than $95 \%$ of the users. Edge users will experience ICI from a larger number of cells due to a higher reuse factor. This results in a lower SINR as compared to center users and is shown in Figure 6 to be $>0$ $\mathrm{dB}$ more than $95 \%$ of the users in that region. This shows that edge users with a higher reuse factor $(\mathrm{RF}=3)$ will experience worst SINR as compared to center users with a lower reuse

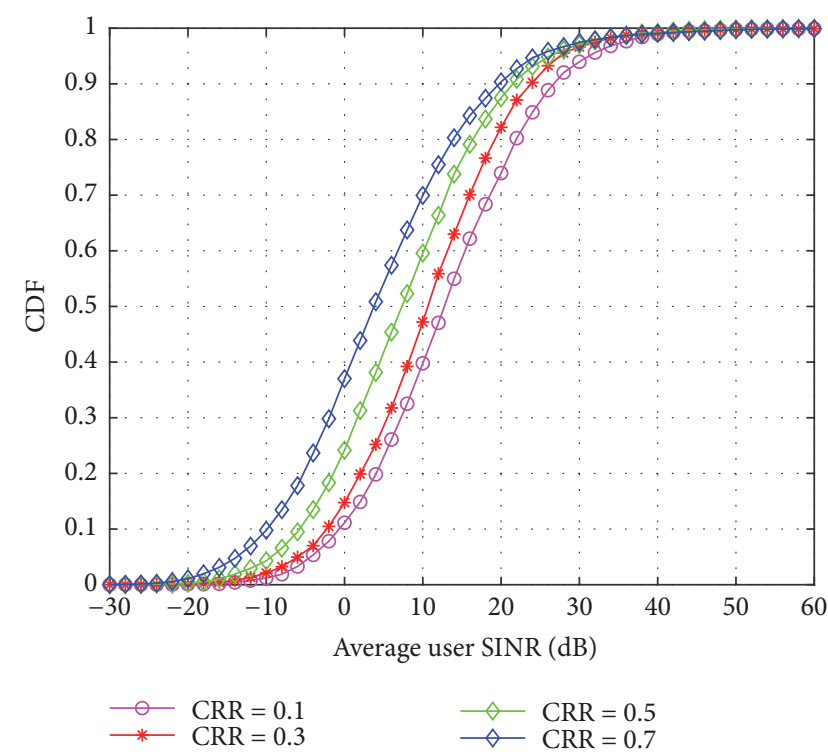

FIGURE 7: Center ratio analysis of user SNR.

factor $(\mathrm{RF}=1)$. This also provides a solid basis for NOMA clustering due to a significant difference in SINRs of users in both cell zones.

5.2.3. Dynamic Fair NOMA FFR. FFR performance depends on how well the cell zone division has been performed as well as the amount of power has been allocated to each user group. Edge users will be allocated more power as per the NOMA requirement, considering the channel degradation due to a larger distance from cell center degradation due to a larger distance from cell center and associated path losses. Two parameters have been defined for analyzing the proposed design: (i) center power ratio (CPR) that is the ratio of power allocated to center users to total transmit power; (ii) Center radius ratio (CRR) that is the ratio of the radius of the central zone to cell radius; (iii) edge radius ratio (ERR), which is the ratio of edge radius and cell radius. Figure 7 plots the SINR distribution of users with different center radius ratios. When CRR $=0.1$, the central region of the cell is very small as compared to the edge region. This will cause a majority of users (about 97\%) to have an SINR value greater than or equal to $-10 \mathrm{~dB}$. As the center radius value is increased, more users will be distributed in a relatively larger center zone causing better SINR values for users. This can be seen for CRR $=0.7$ cases in which about the same number of users have SINR values greater than or equal to a much lower value of $-20 \mathrm{~dB}$.

Figures 8 and 9 depict the throughput values for users for different power ratios as the central radius ratio is altered. For each CPR value, simulations are performed for different values of CRR to obtain the throughput behavior of the center and edge user groups along with their cumulative sum. For center group users, the throughput will increase with an increase in CRR as the number of users in the central region will increase and more channels will be allocated to central users. Interestingly, a decrease in throughput is observed as we cross the half-radius threshold of the cell 


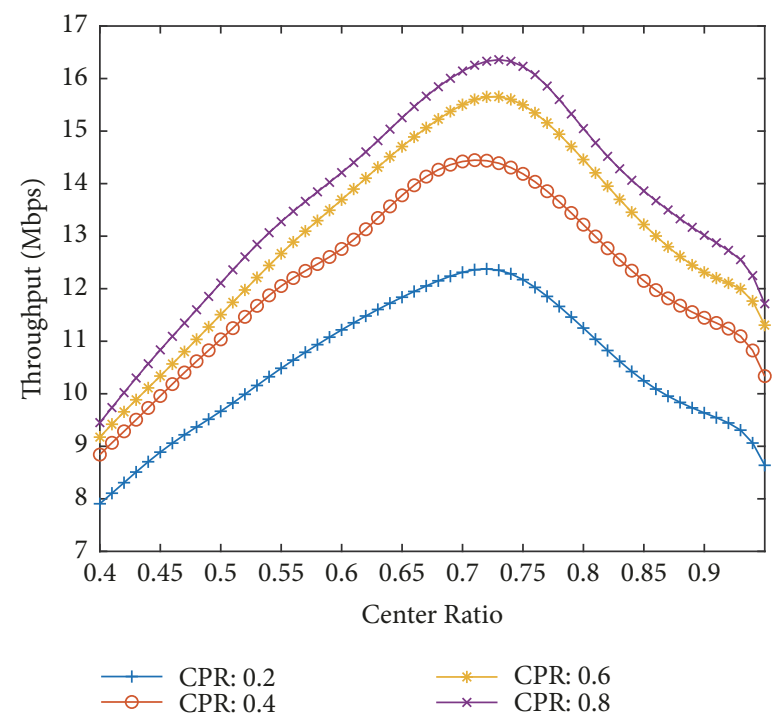

FIGURE 8: CPR impact on center user throughput.

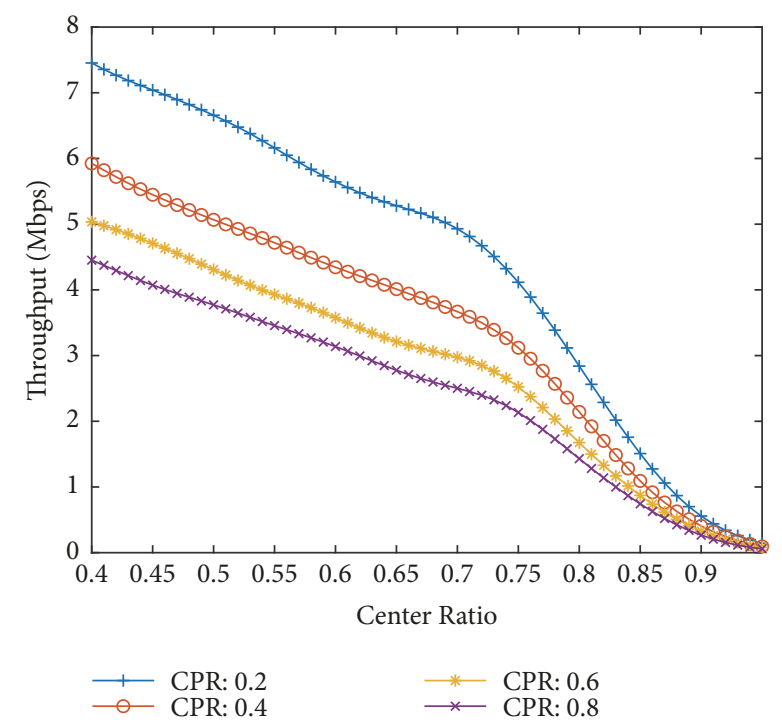

FIGURE 9: CPR impact on edge user throughput.

and increase CRR beyond this point. This is due to the increase in ICI observed by the center users as they are now gradually getting closer to center zones of other cells. Central users are allotted the same frequencies in all cells and ICI cannot be ignored if center zones are greater than the halfcell radius. This is also partially due to the user's fairness criterion due to the imposition of maximum rate limit $R_{\max }$ on center users, which is an integral part of our algorithm. For edge group users, as the values of CRR increase, there is a gradual decrease in overall throughput of edge users, and this is simple to perceive as the central zone is becoming bigger and a larger number of users will be registered as central users. The overall average throughput of edge users will always be declining due to the decreasing number of users in edge zones as compared to the central zones. As per our algorithm, edge users are already on orthogonal frequency

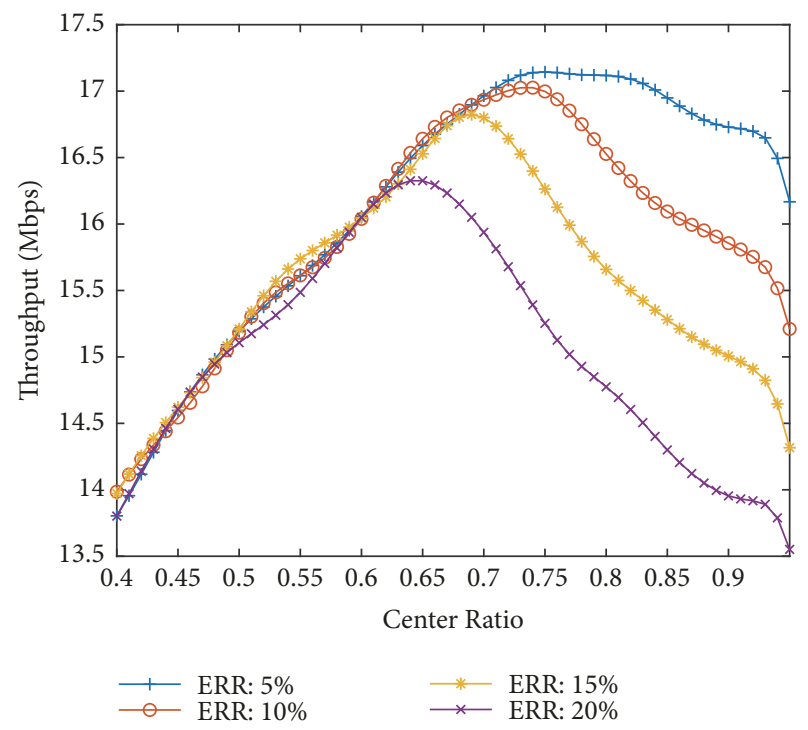

FIGURE 10: ERR impact on center user throughput.

bands with a frequency reuse factor of 3; so, ICI is already reduced by using the FFR technique. We will make sure that edge users receive proper service, which is determined by the minimum rate threshold $R_{\min }$ by allocating appropriate resources consistently.

Figures 10 and 11 show the effects of the changing edge zone radius on user throughputs. Edge zone radius varied between 5 and $20 \%$ of the whole cell radius while observing its impact on user throughputs and affecting factors. For the center group, an increase in user throughputs is observed until it crosses the half-radius limit or the overlap with edge zone starts for different ERR values. Afterward, a decrease is observed due to ICI experienced by central users as well as due to any false classifications of edge and central users incorrectly into the wrong zone due to interzonal overlap between central and edge zones. For the edge group, different ERR values are adopted and CRR is altered to observe user throughput behavior. As the central zone radius of the cell increases, more users are included in the central zone as compared to the edge zone, which will cause a decrease in throughput for edge users. A steeper decline is observed after the specific value of CRR for each ERR value due to the overlap of both edge and center bands, which will lead user false classifications in both bands. For both center and edge users, the fairness criterion is also enforced, respectively, and will also limit the achievable throughput by both user groups and once it has been achieved a decline is observed after that point.

Performance enhancement for NOMA using the proposed algorithm can be clearly identified by comparing cumulative distribution functions (CDF) of both center and edge user groups for different power ratio values. It can be clearly seen in Figure 12 that, by applying the proposed scheme, a significant improvement is observed that has different implications for both center and edge user groups. For center user group, a lower power ratio is required for NOMA implementation to the proposed design as compared 


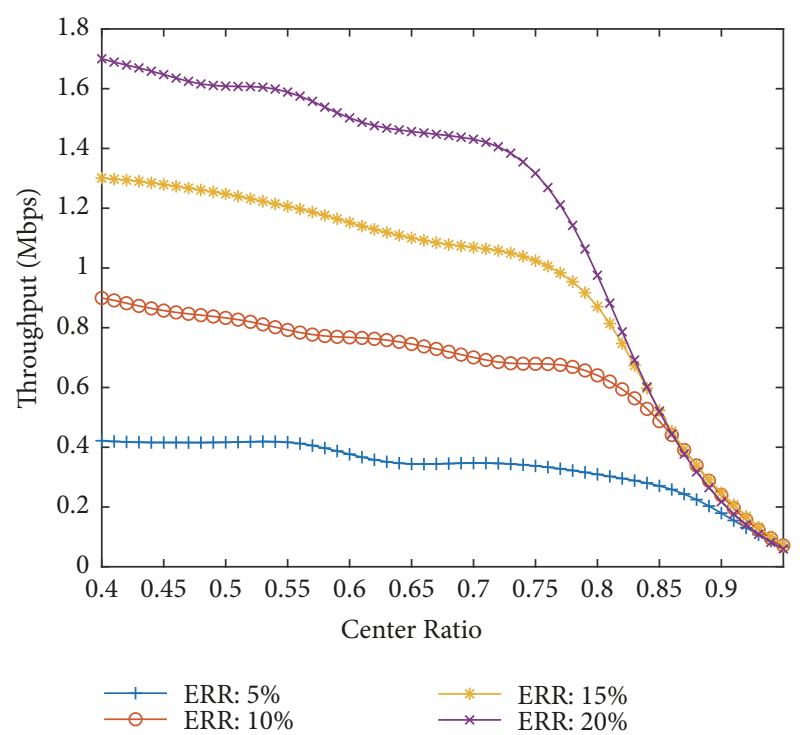

FIgURE 11: ERR impact on edge user throughput.

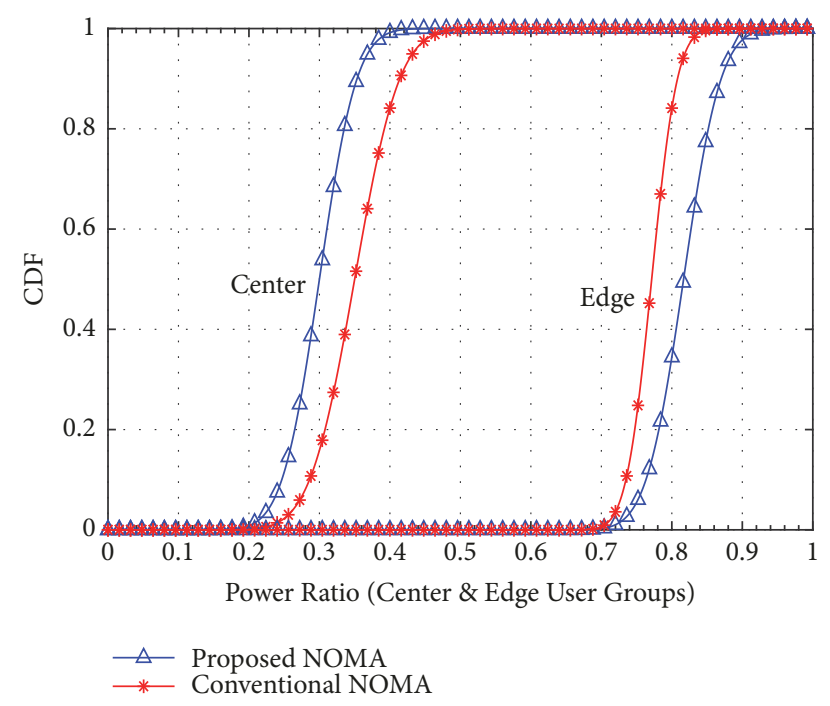

FigURE 12: Power allocation impact on center and edge user groups.

to the conventional NOMA system. Power allocation for edge users in the proposed scheme is greater than conventional NOMA to ensure compensation of the ICI experienced by edge users. CC users will have a higher bandwidth available to them as compared to CE users after the implementation of FFR in the proposed NOMA design, thereby providing CC users more freedom in the frequency domain.

In Figure 13, spectral performance trends of CC and CE users is depicted under different transmission modes and the comparison is performed with proposed and conventional NOMA schemes for benchmarking. No impact is observed for CC users in either of the mentioned schemes with a change in location within the center zone of the cells due to considerably lower levels of ICI. A point to observe here is that our scheme provides just enough (but still higher than OMA) spectral efficiency to center users due to a
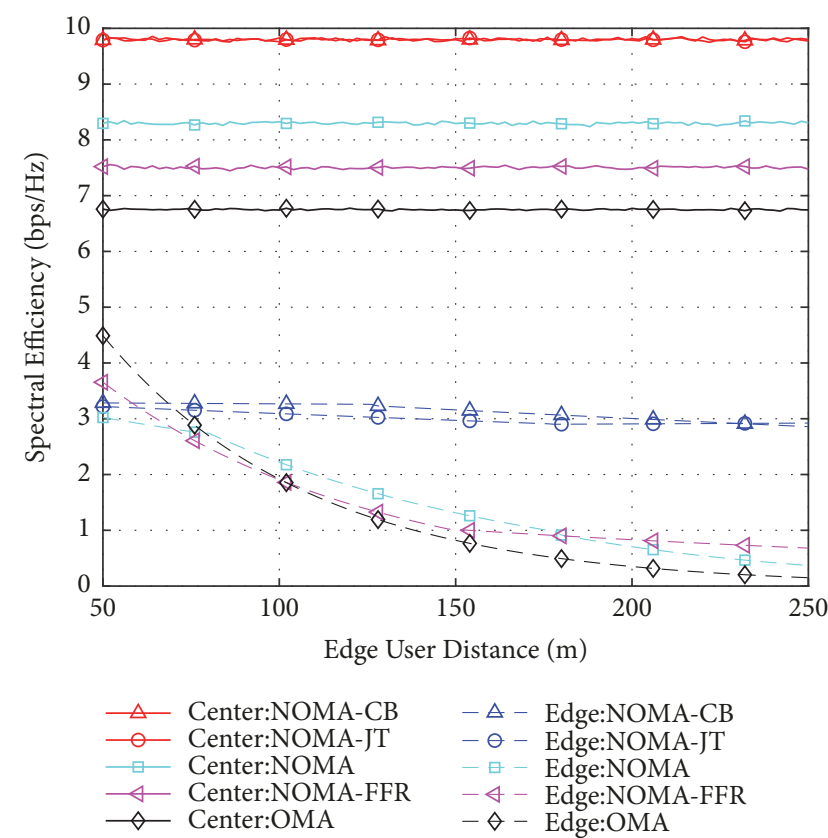

FIGURE 13: Spectral performance comparison of ICI mitigation schemes.

hybrid design and clustering limitations in user pairings (lack of CSI diversity amongst users). Generally, a decrease in performance for OMA and NOMA is observed with the changing location of CE users as no ICI mitigation is applied. NOMA-JT matches the performance of NOMA-CB with an increase in gain as the CE users get closer to the cell boundary because CE user can now take advantage of the link from the neighboring $\mathrm{BS}$ to increase its SINR via data sharing. OMA outperforms all the schemes when CE users are close to $\mathrm{BS}$, mainly due to the remaining interuser interference in all NOMA schemes. Proposed scheme outperforms OMA as well as conventional NOMA schemes in the edge zone of the cell owing to better ICI handling capability as well as low signaling overhead and data sharing requirement as in schemes like NOMA-CB and JT. This provides a rather simplistic ICI mitigation design as compared to $\mathrm{CB}$ and JT based designs due to limited coordination required amongst users, which leads to savings in computational capability and information acquisition design.

In Figure 14, outage performance of the proposed scheme is compared with OMA and available NOMA approaches for handling ICI. Outage performance of proposed scheme can be analyzed by obtaining probability of edge users being in outage from (12) for the proposed scheme. For conventional NOMA and OMA schemes, outage probabilities were approximated as shown in detail by Oviedo [42]. Final formulas have been included for reference in the Appendix. Expectedly, OMA and conventional NOMA design have a higher chance of being in outage due to inability to cater for any experienced ICI by users in edge zone. NOMA$\mathrm{CB}$ requires a complex beamforming and a beam steering mechanism in order to effectively cancel out any effects of ICI on edge users leading to an improved outage performance. 


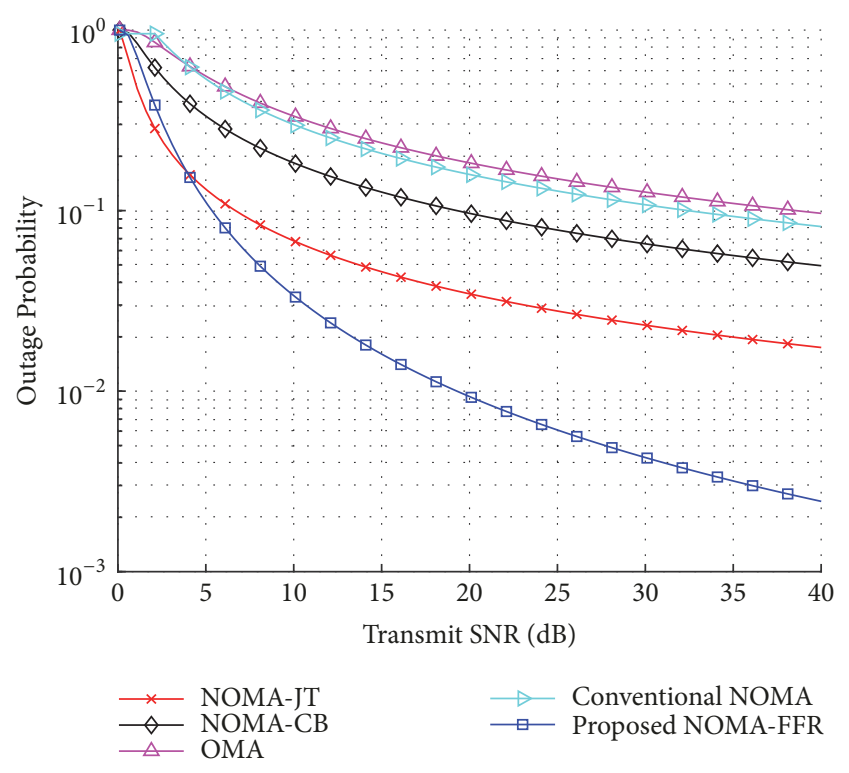

FIGURE 14: Outage performance comparison of CE users.

Similarly, NOMA-JT improves outage performance due to the inherent data sharing in CE users amongst neighboring cells, leading to an improved SINR as transmit power is increased. Lastly, the proposed scheme outperforms all previous schemes by employing cell zoning as well as ICI aware power allocation and user clustering in respective cell zones for CC and CE users. NOMA-FFR suffers in terms of better spectral utilization as compared to other ICI mitigation techniques but makes up for it by improving the interference handling capability of users.

\section{Conclusion}

In this paper, the importance of interference mitigation in the multicellular downlink NOMA design was demonstrated and different possible techniques were discussed that can be used to minimize and isolate ICI to improve edge user performance. A proportional fairness-based channel allocation and power control algorithm were then proposed to achieve ICI minimization by exploiting a rather known technique of FFR. Numerical results indicate that NOMA design with the proposed scheme improves the user performance for both edge and center users. Power allocations have a direct impact on achieving user rates as expected in NOMA as well as compensation of the experienced interference in environment. Effects of an efficient selection of cell zoning with respect to user density were also discussed and it has been emphasized that the selection of cell zoning threshold plays a key role in ensuring service to NOMA users, especially users in the edge zone. Factors that have an impact on ICI in NOMA include power and resource allocation, cell zoning, and a suitable selection of fairness thresholds for edge and center users. ICI can further be minimized by using different modified forms of FFR (e.g., SFR, DFFR) and will be the prime focus of any future enhancements in this work. CoMP techniques can also be used for cell edge, so that interference effects can be minimized by mutual information sharing. SIC error minimization for NOMA is another potential area of research that can be exploited for enhancing intracluster performance in NOMA.

\section{Appendix}

\section{Outage Probability of NOMA and OMA}

Outage performance analysis has already been performed for NOMA and OMA systems in previous works like Oviedo [42], which has been utilized in this paper for comparison purposes. According to channel gain information $\left(\left|h_{2}\right|>\right.$ $\left.\left|h_{1}\right|\right), U E_{1}$ and $U E_{2}$ will be present in the edge and center region of the cell, respectively. Power allocation to $U E_{1}$ will be more to compensate for low channel gain and vice versa.

Here, outage probability expressions have been presented from [42] for OMA users that are given with respect to channel gain experienced within a cluster by users in different cell zones.

$$
\begin{aligned}
P_{1}^{O M A} & =1-\exp \left[\frac{-2\left(4^{R_{\text {min }}}-1\right)}{\beta P_{\max }}\right] \\
P_{2}^{O M A} & =1+\exp \left[\frac{-2\left(4^{R_{\min }}-1\right)}{\beta P_{\max }}\right] \\
& -2 \exp \left[\frac{-2\left(4^{R_{\min }}-1\right)}{\beta P_{\max }}\right]
\end{aligned}
$$

For NOMA users, outage probability can be found in a similar way by following expressions,

$$
\begin{aligned}
P_{1}^{N O M A}= & +\exp \left[\frac{-\alpha_{2}}{\beta}\right] \\
& -\frac{2}{\beta} \int_{\alpha_{2}}^{\infty} \exp \left[\frac{-x\left(\alpha_{1}+1\right)}{\beta}\right] d x
\end{aligned}
$$

where

$$
\begin{aligned}
& \alpha_{1}=\frac{2^{R_{\min }}-1}{x P_{\max }+2^{R_{\min }}\left(1-\sqrt{1+x P_{\max }}\right)} \\
& \alpha_{2}=\frac{4^{R_{\text {min }}}-2}{2 P_{\max }}+\sqrt{\frac{4^{R_{\min }}-1}{2 P_{\max }^{2}}+\frac{\left(4^{R_{\text {min }}}-2\right)^{2}}{4 P_{\max }^{2}}} \\
& P_{2}^{N O M A}=1+\exp \left[\frac{-2\left(4^{R_{\text {min }}}-1\right)}{\beta P_{\max }}\right]-2 \\
& \cdot \exp \left[\frac{-2\left(2^{R_{\min }}-1\right)}{\beta P_{\max }}\right]+\left(2^{R_{\min }}-1\right) \text {. } \\
& \exp \left[\frac{\left(2^{R_{\min }}-3\right)^{2}}{4 \beta P_{\max }}\right] \cdot \sqrt{\frac{\pi}{\beta P_{\max }}} \text {. } \\
& {\left[\operatorname{erf} c\left\langle\frac{\left(2^{R_{\text {min }}}+1\right)}{2 \sqrt{\beta P_{\text {max }}}}\right\rangle-\operatorname{erf} c\left\langle\frac{\left(6^{R_{\text {min }}}+1\right)}{2 \sqrt{\beta P_{\text {max }}}}\right\rangle\right]}
\end{aligned}
$$


Proof. See [42], Appendix C.

Average outage probabilities have been calculated by considering $\beta=1$ for all the users in a particular region of the cell.

\section{Data Availability}

The data used to support the findings of this study are available from the corresponding author upon request.

\section{Conflicts of Interest}

The authors declare that they have no conflicts of interest.

\section{Acknowledgments}

The National Research Foundation of Korea (NRF) grant funded by the Korea government (MSIP) (2016R1A2B4008457) and the Strengthening R \& D Capability Program of Sejong University supported this work.

\section{References}

[1] M. S. Ali, H. Tabassum, and E. Hossain, "Dynamic User Clustering and Power Allocation for Uplink and Downlink NonOrthogonal Multiple Access (NOMA) Systems," IEEE Access, vol. 4, pp. 6325-6343, 2016.

[2] 3GPP TSG-RAN R1-050738, "FFR, Interference mitigation considerations and results on frequency reuse," September 2005.

[3] J. Choi, "Non-orthogonal multiple access in downlink coordinated two-point systems," IEEE Communications Letters, vol. 18, no. 2, pp. 313-316, 2014.

[4] S. M. Alamouti, "A simple transmit diversity technique for wireless communications," IEEE Journal on Selected Areas in Communications, vol. 16, no. 8, pp. 1451-1458, 1998.

[5] S. Han, Chih-Lin I, Z. Xu, and Q. Sun, "Energy Efficiency and Spectrum Efficiency Co-Design: From NOMA to Network NOMA," IEEE MMTC E-Letter, vol. 9, no. 5, pp. 21-24, 2014.

[6] D. Lee, H. Seo, B. Clerckx et al., "Coordinated multipoint transmission and reception in LTE-advanced: deployment scenarios and operational challenges," IEEE Communications Magazine, vol. 50, no. 2, pp. 148-155, 2012.

[7] L. Ping, L. Liu, K. Wu, and W. K. Leung, "Interleave-division multiple-access," IEEE Transactions on Wireless Communications, vol. 5, no. 4, pp. 938-947, 2006.

[8] R. Hoshyar, F. P. Wathan, and R. Tafazolli, "Novel low-density signature for synchronous CDMA systems over AWGN channel," IEEE Transactions on Signal Processing, vol. 56, no. 4, pp. 1616-1626, 2008.

[9] X. Dai, S. Chen, S. Sun et al., "Successive interference cancelation amenable multiple access (SAMA) for future wireless communications," in Proceedings of the 2014 IEEE International Conference on Communication Systems, IEEE ICCS 2014, pp. 222-226, China, November 2014.

[10] S. Chen, B. Ren, Q. Gao, S. Kang, S. Sun, and K. Niu, "Pattern division multiple access-a novel nonorthogonal multiple access for fifth-generation radio networks," IEEE Transactions on Vehicular Technology, vol. 66, no. 4, pp. 3185-3196, 2017.
[11] B. Ren, X. Yue, W. Tang et al., "Advanced IDD receiver for PDMA uplink system," in Proceedings of the 2016 IEEE/CIC International Conference on Communications in China, ICCC 2016, China, July 2016.

[12] J. Zeng, B. Li, X. Su, L. Rong, and R. Xing, "Pattern division multiple access (PDMA) for cellular future radio access," in Proceedings of the 2015 International Conference on Wireless Communications \& Signal Processing (WCSP), pp. 1-5, Nanjing, China, October 2015.

[13] B. Ren, Y. Wang, X. Dai, K. Niu, and W. Tang, "Pattern matrix design of PDMA for 5G UL applications," China Communications, vol. 13, pp. 159-173, 2016.

[14] P. Li, Y. Jiang, S. Kang et al., "Joint Transmitter and Receiver Design for Spatial Pattern Division Multiple Access with Largescale Antenna," in Proceedings of the 65 China Communications ¿amp; Supplement No. 22016 submitted for publication, PIMRC2016, oint Transmitter and Receiver Design for Spatial Pattern Division Multiple Access with Large-scale Antenna, Ed., April 2016.

[15] H. Nikopour and H. Baligh, "Sparse code multiple access," in Proceedings of the IEEE 24th Annual International Symposium on Personal, Indoor, and Mobile Radio Communications (PIMRC '13), pp. 332-336, IEEE, London, UK, September 2013.

[16] M. Taherzadeh, H. Nikopour, A. Bayesteh, and H. Baligh, "SCMA codebook design," in Proceedings of the 80th IEEE Vehicular Technology Conference, VTC 2014-Fall, Canada, September 2014.

[17] 3GPP, "Huawei, HiSilicon, Sparse Code Multiple Access (SCMA) for 5G Radio Transmission," R1- 162155, April 2016.

[18] M. Al-Imari, P. Xiao, M. A. Imran et al., "Uplink NonOrthogonal Multiple Access for 5G Wireless Networks," in Proceedings of the ISWCS 2014, 2014.

[19] 3GPP, "ZTE, Discussion on multiple access for new radio interface," R1-162226, April 2016.

[20] Z. Yuan, G. Yu, W. Li, Y. Yuan, X. Wang, and J. Xu, "Multi-User Shared Access for Internet of Things," in Proceedings of the 2016 IEEE 83rd Vehicular Technology Conference (VTC Spring), pp. 1-5, Nanjing, China, May 2016.

[21] H. Jin, K. Peng, and J. Song, "Bit division multiplexing for broadcasting," IEEE Transactions on Broadcasting, vol. 59, no. 3, pp. 539-547, 2013.

[22] White paper, "v2.0D-Alternative Multiple access vl," November 2015, http://www.future-forum.org/zhuanti/151105/cn/index .asp.

[23] 3GPP, Qualcomm Inc., Candidate NR multiple access schemes, R1-162202, Apr. 2016.

[24] 3GPP, Qualcomm Inc., RSMA, R1- 164688, May 2016.

[25] 3GPP, "Samsung, Non-Orthogonal Multiple Access Candidate for NR," R1-163992, May 2016.

[26] A. Li, Y. Lan, X. Chen, and H. Jiang, "Non-orthogonal multiple access (NOMA) for future downlink radio access of 5G," China Communications, vol. 12, pp. 28-37, 2015.

[27] K. Higuchi and Y. Kishiyama, "Non-orthogonal access with random beamforming and intra-beam SIC for cellular MIMO downlink," in Proceedings of the 2013 IEEE 78th Vehicular Technology Conference, VTC Fall 2013, USA, September 2013.

[28] K. Higuchi and A. Benjebbour, "Non-Orthogonal Multiple Access (NOMA) with successive interference cancellation for future radio access," IEICE Transactions on Communications, vol. E98B, no. 3, pp. 403-414, 2015. 
[29] 3GPP, "Study on Downlink Multiuser Superposition Transmission (MUST) for LTE (Release 13),” TR36.859, December2015.

[30] 3GPP, "MediaTek Inc., CMCC, etc. New work item proposal: Downlink Multiuser Superposition Transmission for LTE," RP160680, March 2016.

[31] 3GPP, "NTT-DOCOMO, Initial views and evaluation result on non-orthogonal multiple access for NR uplink," R1-163111, April 2016.

[32] 3GPP, "NTT-DOCOMO, Initial views and evaluation results on non-orthogonal multiple access for NR,” R1-165175, May 2016.

[33] 3GPP TSG-RAN R1-050507, "SFR, Soft frequency reuse scheme for UTRAN LTE”, 3GPP, May 2005.

[34] Y. Umeda and K. Higuchi, "Efficient adaptive frequency partitioning in OFDMA downlink with fractional frequency reuse," in Proceedings of the 2011 International Symposium on Intelligent Signal Processing and Communications Systems (ISPACS 2011), pp. 1-5, Chiang Mai, Thailand, December 2011.

[35] W. Shin, M. Vaezi, B. Lee, D. J. Love, J. Lee, and H. V. Poor, "Coordinated beamforming for multi-cell MIMO-NOMA," IEEE Communications Letters, vol. 21, no. 1, pp. 84-87, 2017.

[36] Z. Ding, F. Adachi, and H. V. Poor, "The Application of MIMO to Non-Orthogonal Multiple Access," IEEE Transactions on Wireless Communications, vol. 15, no. 1, pp. 537-552, 2016.

[37] N. Saquib, E. Hossain, and D. I. Kim, "Fractional frequency reuse for interference management in LTE-advanced hetnets," IEEE Wireless Communications Magazine, vol. 20, no. 2, pp. 113122, 2013.

[38] T. D. Novlan, R. K. Ganti, A. Ghosh, and J. G. Andrews, "Analytical evaluation of fractional frequency reuse for OFDMA cellular networks," IEEE Transactions on Wireless Communications, vol. 10, no. 12, pp. 4294-4305, 2011.

[39] T. Novlan, J. G. Andrews, I. Sohn, R. K. Ganti, and A. Ghosh, "Comparison of fractional frequency reuse approaches in the OFDMA cellular downlink," in Proceedings of the 53rd IEEE Global Communications Conference, (GLOBECOM '10), pp. 1-5, Miami, Fla, USA, December 2010.

[40] Zubin Bharucha and Harald Haas, "The Distribution of Path Losses for Uniformly Distributed Nodes in a Circle," Research Letters in Communications, vol. 2008, pp. 1-4, 2008.

[41] N. Otao, Y. Kishiyama, and K. Higuchi, "Performance of nonorthogonal access with SIC in cellular downlink using proportional fair-based resource allocation," in Proceedings of the 2012 9th International Symposium on Wireless Communication Systems, ISWCS 2012, pp. 476-480, August 2012.

[42] J. A. Oviedo and H. R. Sadjadpour, "A Fair Power Allocation Approach to NOMA in Multiuser SISO Systems," IEEE Transactions on Vehicular Technology, vol. 66, no. 9, pp. 7974-7985, 2017. 


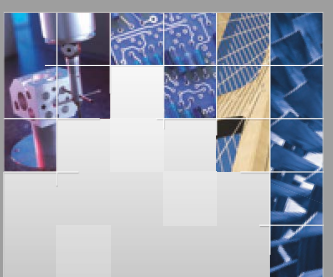

\section{Enfincering}
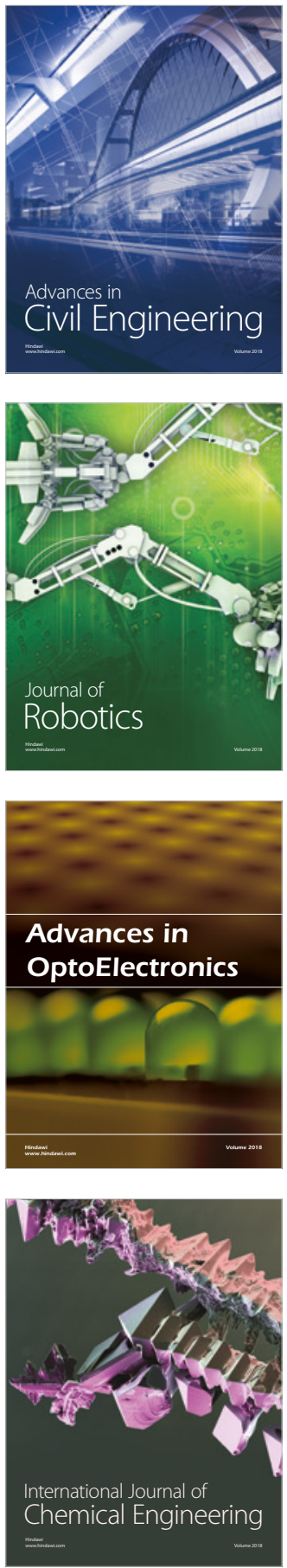

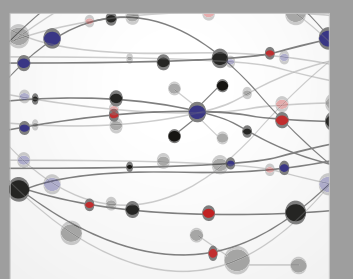

\section{Rotating \\ Machinery}

The Scientific World Journal

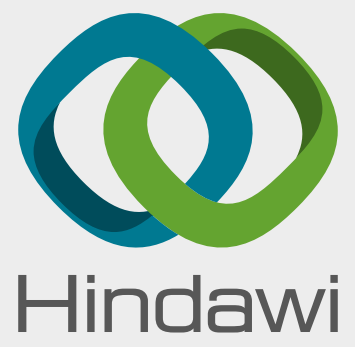

Submit your manuscripts at

www.hindawi.com
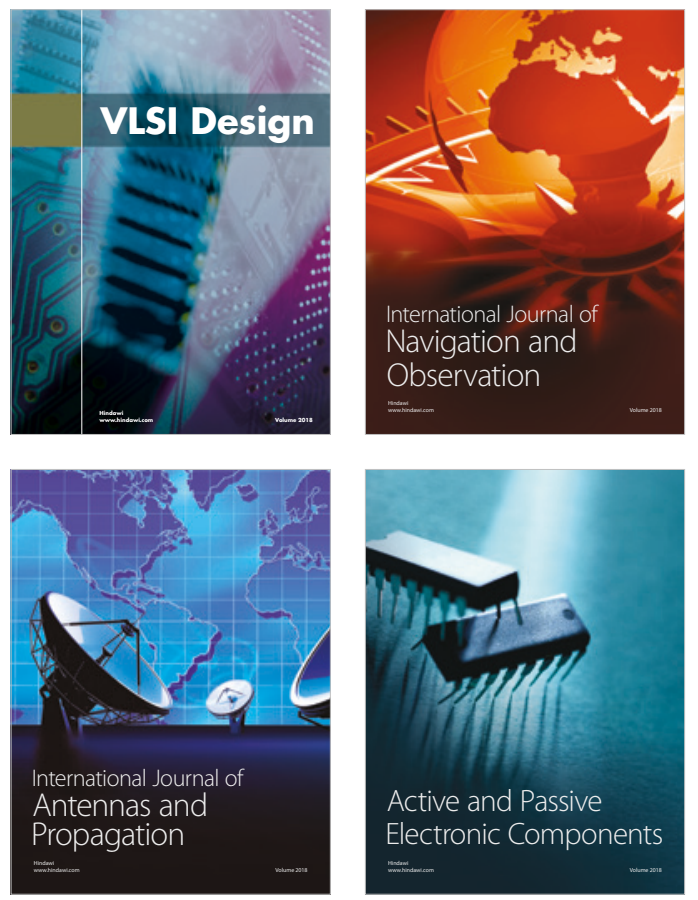
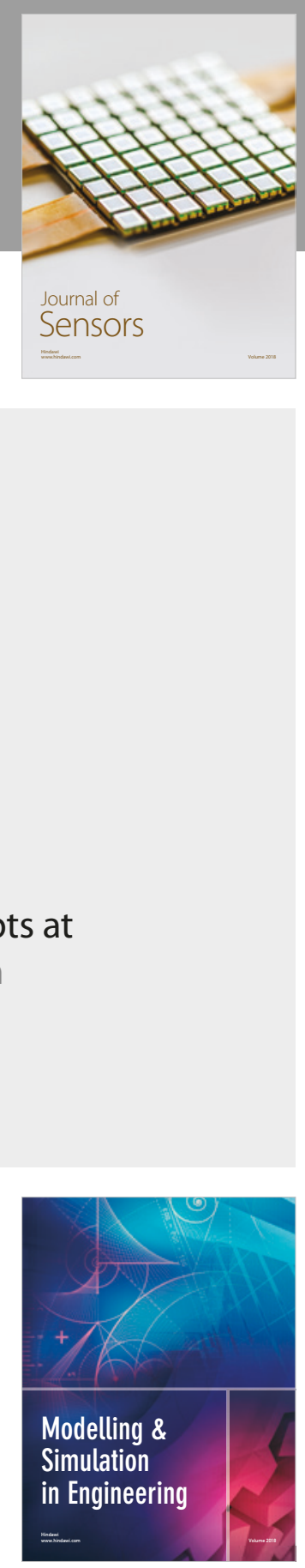

\section{Advances \\ Multimedia}
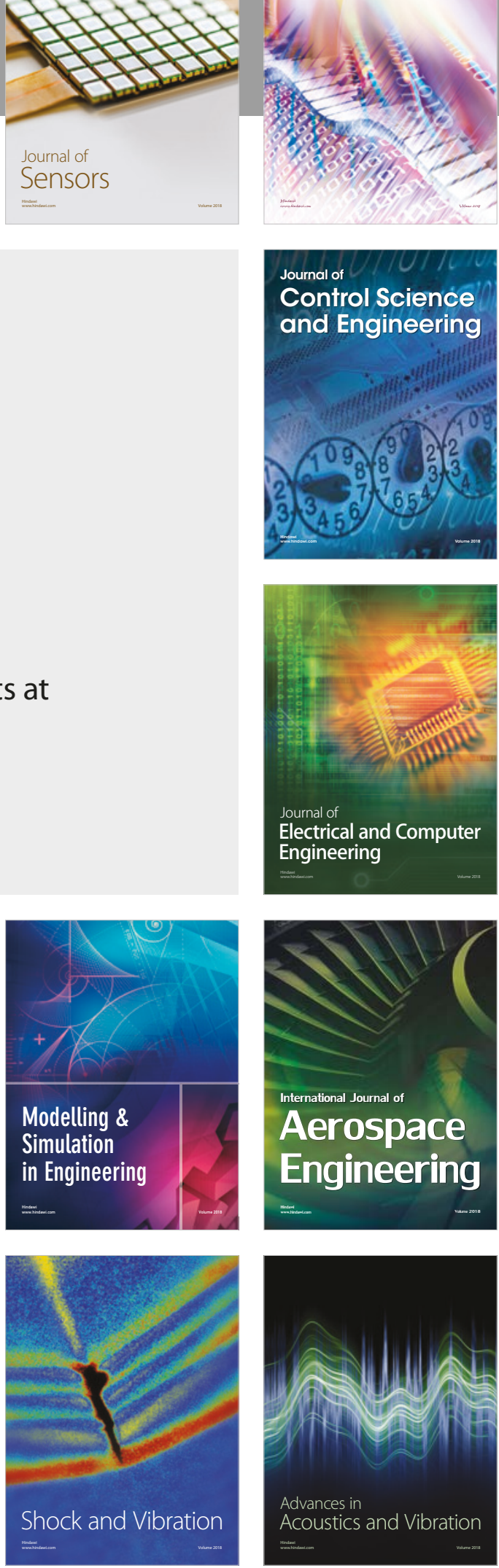